Silverii, F., Maccaferri, F., Richter, G., Gonzalez

Cansado, B., Wang, R., Hainzl, S., Dahm, T. (2021):

Poroelastic model in a vertically sealed gas

storage: a case study from cyclic injection/

production in a carbonate aquifer. - Geophysical

Journal International, 227, 2, 1322-1338.

https://doi.org/10.1093/gji/ggab268 


\title{
Poroelastic model in a vertically sealed gas storage: a case study from cyclic injection/production in a carbonate aquifer
}

\author{
F. Silverii ${ }^{\oplus},{ }^{1}$ F. Maccaferri, ${ }^{1,2}$ G. Richter, ${ }^{1,3}$ B. Gonzalez Cansado, ${ }^{4}$ R. Wang, ${ }^{1,5}$ \\ S. Hainzl ${ }^{1}$ and T. Dahm ${ }^{\oplus 1,6}$ \\ ${ }^{1}$ Physics of Earthquakes and Volcanoes, German Research Centre for Geosciences (GFZ), Potsdam 14467, Germany. E-mail: silverii@gfz-potsdam.de \\ ${ }^{2}$ National Institute of Geophysics and Volcanology (INGV), Osservatorio Vesuviano, Napoli 80124, Italy \\ ${ }^{3}$ Institute of Mathematics, University of Potsdam, Potsdam 14476, Germany \\ ${ }^{4}$ Enagás S.A.U., Madrid 28005, Spain \\ ${ }^{5}$ State Key Laboratory of Earthquake Dynamics, Institute of Geology, China Earthquake Administration, Beijing 100029, China \\ ${ }^{6}$ Institute of Geosciences, University of Potsdam, Potsdam 14476, Germany
}

Accepted 2021 July 2. Received 2021 July 2; in original form 2021 April 6

\begin{abstract}
S UMMAR Y
Natural gas can be temporarily stored in a variety of underground facilities, such as depleted gas and oil fields, natural aquifers and caverns in salt rocks. Being extensively monitored during operations, these systems provide a favourable opportunity to investigate how pressure varies in time and space and possibly induces/triggers earthquakes on nearby faults. Elaborate and detailed numerical modelling techniques are often applied to study gas reservoirs. Here we show the possibilities and discuss the limitations of a flexible and easily formulated tool that can be straightforwardly applied to simulate temporal pore-pressure variations and study the relation with recorded microseismic events. We use the software POEL (POroELastic diffusion and deformation) which computes the poroelastic response to fluid injection/extraction in a horizontally layered poroelastic structure. We further develop its application to address the presence of vertical impermeable faults bounding the reservoir and of multiple injection/extraction sources. Exploiting available information on the reservoir geometry and physical parameters, and records of injection/extraction rates for a gas reservoir in southern Europe, we perform an extensive parametric study considering different model configurations. Comparing modelled spatiotemporal pore-pressure variations with in situ measurements, we show that the inclusion of vertical impermeable faults provides an improvement in reproducing the observations and results in pore-pressure accumulation near the faults and in a variation of the temporal porepressure diffusion pattern. To study the relation between gas storage activity and recorded local microseismicity, we applied different seismicity models based on the estimated porepressure distribution. This analysis helps to understand the spatial distribution of seismicity and its temporal modulation. The results show that the observed microseismicity could be partly linked to the storage activity, but the contribution of tectonic background seismicity cannot be excluded.
\end{abstract}

Key words: Permeability and porosity; Gas and hydrate systems; Europe; Induced seismicity.

\section{INTRODUCTION}

In order to manage seasonal fluctuations in energy consumption against a relatively stable production capacity, buffering techniques have been developed. This concept also applies to natural gas, typically characterized by higher demand in winter. A common way to deal with demand fluctuations, and take advantage of marketprice variations, is to store natural gas in Underground Gas Storage (UGS) facilities, such as depleted gas and oil fields, natural aquifers and caverns (natural or excavated) in salt rocks (e.g. Liu et al. 2018;
Wu et al. 2020). In particular, underground natural aquifers consisting of porous and permeable rock formations act as natural water reservoirs and, in some cases, can be used for natural gas storage. As of 2018, there were more than 600 underground natural gas storage facilities worldwide, $\sim 80$ of which are in deep saline aquifers, the first dating back to 1925 (Cornot-Gandolphe, CEDIGAZ November 2018 Report, https://cdn2.hubspot.net/hubfs/1982707/Overvie $\mathrm{w}$ of underground gas storage in the world 2018 (1).pdf).

Underground operations causing perturbations of the stress field or pore-pressure can potentially modulate seismicity. In recent 
years, seismicity induced and triggered by human activities has become an important topic of interest. Earthquakes have been observed as a consequence of a wide range of human activities such as fluid or gas injection/extraction into underground formations, mining or quarry sites, and fluid impoundment of reservoirs (e.g. Shapiro et al. 2006; Evans et al. 2012; Grünthal 2014; Foulger et al. 2018; Richter et al. 2020). However, attributing the cause of an earthquake to human activity and discriminating between anthropogenic and natural seismicity is not straightforward (Grigoli et al. 2017).

To minimize the risks related to UGS operations, industries and/or public institutions (depending on specific state regulations) monitor signals such as surface deformation, pore-pressure variation and microseismicity during storage and withdrawal (production) of gas. When carefully monitored, these observations allow to investigate how pressure in reservoirs evolves, modifies the surrounding stressstate, produces deformation coupled with diffusion processes, and possibly induces/triggers earthquakes on nearby faults (e.g. Improta et al. 2015).

In this context, our main objective is to use a flexible, semianalytical poroelastic model to simulate temporal variations of porepressure and study the interaction between pore-pressure diffusion processes and surrounding faults, addressing a possible relation with observed microseismicity.

The study of poroelastic response to fluid injection/extraction in underground reservoirs, and in particular of the pore-pressure diffusion, is usually performed using either analytical, semi-analytical or numerical approaches (e.g. Wang \& Kümpel 2003; Ricard et al. 2012; Altmann et al. 2010, 2014; Chang \& Segall 2016, 2017; Goebel et al. 2017).

Fully analytical models are generally easy and fast to implement. However, analytical solutions for coupled poroelastic problems are only known for very simple configurations, for example for homogeneous isotropic poroelastic media. On the other end, fully numerical simulations, such as finite element or finite difference approaches, usually provide more realistic models being able to account for several complexities, such as material heterogeneity and anisotropy, lateral heterogeneities due to the presence of faults, two-phase flow, complexities of the production/injection history and full 3-D evolution of the pressure field. However, the numerical models are usually more laborious to implement and can require high computing capability and the knowledge of several a priori information about the study area. This could, in turn, pose limitations for the model domain extension and/or its spatiotemporal resolution. Additionally, exploiting the full parameter space or Bayesian and bootstrapping approaches to estimate model uncertainties are difficult to realize with full numerical simulations.

Semi-analytical approaches can represent a valid alternative method when analytical solutions are not available, such as when dealing with a layered half-space or when using the numerical procedures mentioned above is challenging (e.g. Wang \& Kümpel 2003; Goebel et al. 2017).

Here we use the software POEL (POroELastic diffusion and deformation) by Wang \& Kümpel (2003), a semi-analytical physicsbased numerical scheme that allows the computation of transient and steady-state fully coupled poroelastic solutions associated with injection/extraction of fluids in point or extended sources embedded in a horizontally layered poroelastic half-space. POEL is particularly suitable to link observables such as pore-pressure and Coulomb stress variations within the underground reservoir with the physi$\mathrm{cal} / \mathrm{mechanical}$ processes occurring in the surroundings. Therefore, it has been widely used to investigate the relation between fluid injection, pore-pressure diffusion, surface deformation, and induced seismicity in several study areas such as the super-deep drilling project KTB in Germany (Jahr et al. 2005, 2006), the vast oil and gas field in Oklahoma, United States (Barbour et al. 2017; Zhai et al. 2019; Deng et al. 2020) and the Montney Basin in British Columbia, Canada (Yu et al. 2019).

Providing only axisymmetric solutions, POEL, however, drastically simplifies the geometry of the reservoir. Focusing on porepressure diffusion, we therefore further implemented the POEL application to address common, relevant problems for gas storage in poroelastic layers: the presence of vertical impermeable faults bounding the reservoir and the presence of multiple, simultaneously active injection/extraction sources. The presence of quasi-vertical faults, such as of graben type or antithetic type, is indeed common in reservoir geology all over the world (e.g. Orlic 2016; Haug et al. 2018; O'Neill et al. 2018). Representing a lateral barrier to fluid flow, these faults can severely separate pressure and fluids into compartments inside the reservoirs and influence the temporal behaviour and spatial distribution of pressure diffusion. Accounting for these structures when modelling reservoir production/injection features can be therefore important to properly investigate the reservoir behaviour and understand the observed signals (e.g. Jolley et al. 2007, 2010; Pasala et al. 2013; Zbinden et al. 2017; Kettermann et al. 2020).

Through a collaboration with a midstream company in southern Europe, we tested this application by studying the temporal variations and spatial distribution of pore-pressure measurements at one of their UGS facilities. Taking advantage of the available information about the reservoir, injection and production-rate records, and pore-pressure measurements (Section 2.1), we performed an extensive analysis considering different model configurations and values of material parameters in order to evaluate the sensitivity of the results and the effect of the impermeable faults (Sections 3.1, 3.2 and 4.1). We then used the estimated pore-pressure spatiotemporal distribution to investigate the possible relation between the injection/extraction activity and the microseismicity recorded in the area (Sections 2.2, 3.3 and 4.2). Finally, in Section 5, we discuss the main results and summarize the main advantages and limits of the proposed method.

\section{STUDY AREA DESCRIPTION AND AVAILABLE DATA}

\subsection{Setting of the underground storage facility (UGS)}

Our case study is an underground storage facility located in southern Europe, where natural gas is stored in a water-saturated, saline aquifer made of porous rock. The aquifer layer, located at a depth of $\sim 2300 \mathrm{~m}$ and made of dolomitic carbonate rock, is sealed above and below by impermeable rock layers (anhydrite) (Fig. 1a).

The facility of interest is managed by a midstream company in southern Europe operating two additional UGS sites. Details about the study region and data sources are not disclosed due to the company confidentiality policy. Cushion gas (i.e. the gas volume necessary to ensure the minimum storage pressure required for optimal gas injection and withdrawal) and working gas (i.e. the gas volume which can be stored or withdrawn at any time in addition to the cushion gas) have been progressively injected in the saline aquifer layer since 2012. Since this date, the facility performs a seasonal operation. In summer (April 1st to October 31st), the injection phase takes place: compressed gas is injected through the 
(a)

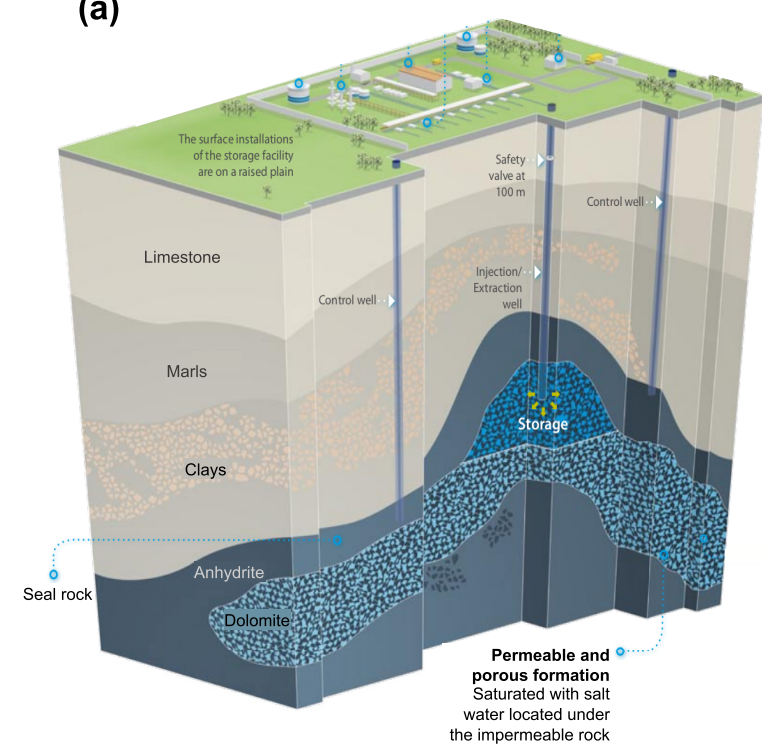

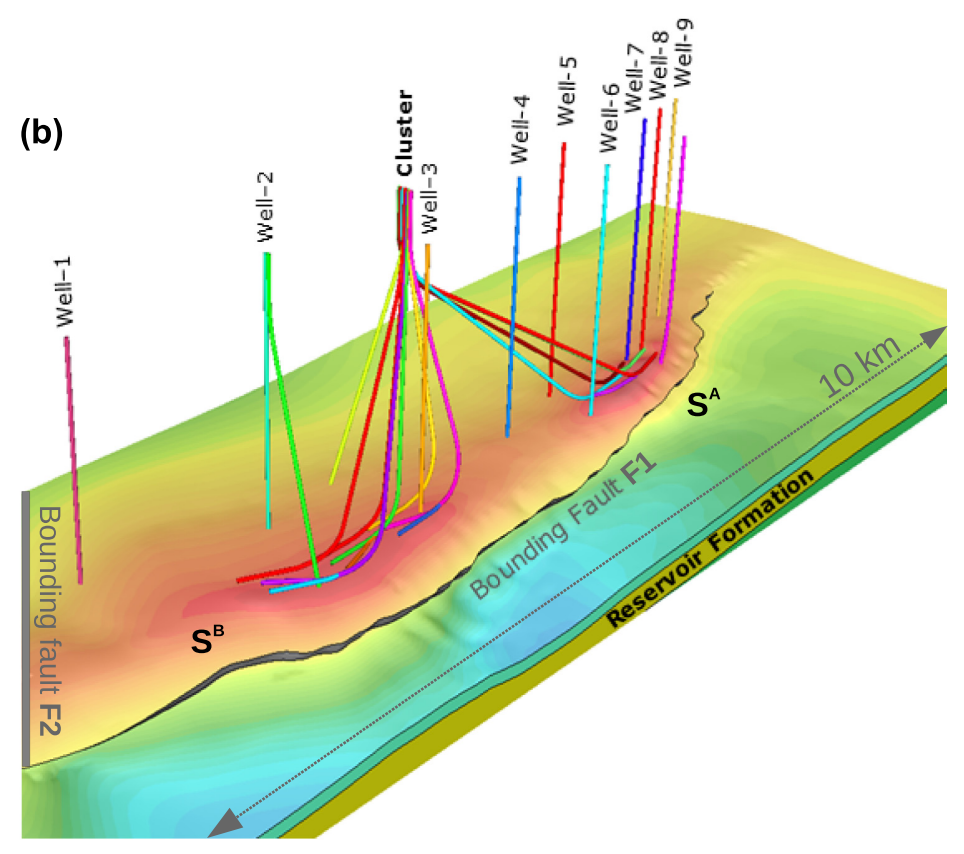

Figure 1. Sketch of the storage facility configuration. (a) Vertical stratification highlighting the porous reservoir layer and the surrounding sealing layers. (b) Map view of the reservoir layer and the injection/production and monitoring wells. $\mathrm{S}^{\mathrm{A}}$ and $\mathrm{S}^{\mathrm{B}}$ labels refer to the two main injection areas, F1 and F2 labels refer to the two main faults that laterally confine the reservoir. The colours are indicative of the depths (isobaths) of the top of the reservoir layer.

wells, displaces the water, and fills the pores in the aquifer layer, which acts as a natural deposit. In winter (November 1 st to March 31st) the production phase takes place: gas is extracted, and water refills the pores in the aquifer. The structure enables to balance the differences in consumption between summer and winter and retains a certain amount of working gas as strategic reserve.

The storage facility consists of two main injection/production areas (hereafter defined ' $\mathrm{S}$ ' ' and ' $\mathrm{S}$ '), and the reservoir is laterally bounded by two main faults (hereafter defined ' $F 1$ ' and 'F2'). Fig. 1(b) shows a sketch of a map view of the reservoir layer and the injection/production and monitoring wells.

The company operating the facility has been monitoring the activity since 2012 and has worked out a simplified geomechanical model. Records of injection/production rates are complemented by pore-pressure measurements and a local seismic network monitoring the microseismicity in the reservoir area. For our research, the company provided: (i) the reservoir geometry (aquifer depth, thickness, and location of the bounding faults), and the approximate dimensions and location of the volume of porous rock containing the cushion gas volume relative to $\mathrm{S}^{\mathrm{A}}$ and $\mathrm{S}^{\mathrm{B}}$, which we used to constrain our model geometry (c.f. Section 3.1); (ii) the physical parameters needed to constrain the poroelastic properties of the reservoir layer and cap rocks, which we used to perform a parametric study and calibrate our model ( $c f$. Section 3.2); (iii) the daily temporal records of total (i.e. group data from all wells) gas injection/production rates relative to $\mathrm{S}^{\mathrm{A}}$ and $\mathrm{S}^{\mathrm{B}}$, which we used as input parameters for our model; (iv) measurements of bottom-hole pore-pressure at two wells located inside each injection area (hereafter $\mathrm{W}^{\mathrm{A}}$ and $\mathrm{W}^{\mathrm{B}}$ ), which are representative of the pore-pressure values within each injection area and which we used for the parametric study, and for the validation of model results (cf. Sections 3.2 and 4.1) and (v) a catalogue of microseismicity recorded by a local seismic network installed in October 2015 ( $c f$. Section 2.2 for more details). Figs 2(a) and (b) show the time-series of injection/production rates
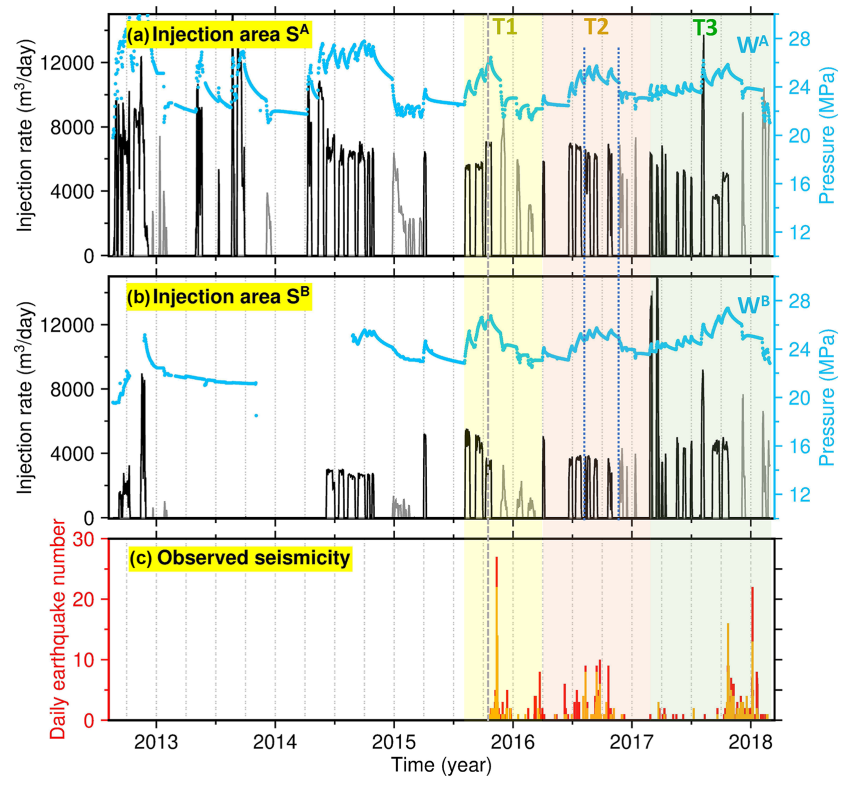

Figure 2. Time-series of injection/production rates (respectively black and grey lines) and pore-pressure (blue dots) at injection area $\mathrm{S}^{\mathrm{A}}$ and well $\mathrm{W}^{\mathrm{A}}$ (a) and injection area $\mathrm{S}^{\mathrm{B}}$ and well $\mathrm{W}^{\mathrm{B}}$ (b). (c) Time-series of observed local seismicity (red bars); the overlapped orange bars indicate the selected seismicity used for the seismicity model. The yellow, orange and green vertical stripes highlight the intervals $\mathrm{T} 1, \mathrm{~T} 2$ and $\mathrm{T} 3$, respectively. The vertical bold-dashed grey line indicates the time when the local seismic network became operational. The blue vertical dotted lines indicate the times referred to in Fig. S4.

and pore-pressure measurements at each injection area. Starting from 2012, several operations of injection/production, each lasting $\sim 10-20 \mathrm{~d}$, have been performed with a seasonal alternation between injection (black lines) and production (grey lines). The measured 
pore-pressure is $\sim 24 \mathrm{MPa}$ with positive (after injection) and negative (after production) variations of $\sim 4 \mathrm{MPa}$ (blue dots).

\subsection{Seismicity data set}

In October 2015, a local seismic network including five stations (Passive Seismic Monitoring Network) was installed, covering an area of roughly $10 \mathrm{~km} \times 10 \mathrm{~km}$ around the facility of interest. Between October 2015 and March 2018, 484 events of small magnitude $(M<2.3)$ have been observed (red bars in Fig. 2c). In particular, the catalog from the local network is characterized by a completeness magnitude of $M_{\mathrm{C}}=0.0$ and consists of 90 per cent microearthquakes $(M<1.0)$ occurring near the UGS $(<25 \mathrm{~km}$ radial distance). The detected seismicity is mostly shallow (95 per cent at depths $\leq 3.5 \mathrm{~km}$, grey bars in Fig. 3a) and spatially clustered. The most prominent cluster occurred close to the bounding fault F2. Fig. 3(b) shows the planar distribution of the observed earthquake density (spatially smoothed by a Gaussian location error with a standard deviation of $1 \mathrm{~km}$ ). This is plotted in the same local metric coordinate system $(x, y)$ used to define the reservoir geometry of the POEL model ( $c f$. Sections 3.1 and 3.3).

The observed seismicity rates vary intensively in time, with three periods of higher seismicity lasting for several weeks (late-2015, 2016.5 to 2017 , late 2017) separated by two periods of very low seismicity. No seismic events are recorded on most days, with a maximum of almost 30 events/day recorded shortly after the network installation in mid-November 2015 (Fig. 2c).

At the location of the UGS there were almost no previous records of seismicity, indicating an overall scarce seismicity of moderate size in the region since 1970 . The national catalogue only includes eight events with $M>2$ between 1970 and 2012 (the starting year of activity at the UGS) in an area of about $30 \mathrm{~km} \times 30 \mathrm{~km}$ around the UGS, and two additional events with $M>2$ since 2012 until 2019 , indicating no significant change of the seismicity rate.

Even if no regional information is available about seismicity with small magnitude $(M \leq 2.0)$ before the installation of the local seismic network, the clustered temporal occurrence of the observed local seismicity (roughly in correspondence with higher recorded pore-pressure values, Fig. 2) and its spatial distribution (shallow depth and mainly clustered near the F2 bounding fault, Fig. 3) possibly suggest a relation with the UGS activity, and motivate our analysis (Sections 3.3 and 4.2).

\section{METHODS}

\subsection{Pore-pressure model: geometry setup and implementation}

In order to model the pore-pressure diffusion in the UGS, we implemented a poroelastic model based on a horizontally layered structure using the software POEL by Wang \& Kümpel (2003). This is a semi-analytical scheme based on an orthonormalization extension of Haskell's propagator method combined with Biot's theory of linear poroelasticity (Biot 1941). It allows the computation of transient and steady-state fully coupled poroelastic solutions in multilayered full and half-space in response to fluid injection/extraction. POEL considers radially unbounded layers and is therefore intrinsically axisymmetric. The model input consists of injection/extraction rates from a fixed source (i.e. source position and dimensions do not vary in time) that can be either punctual, linear or volumetric (cylindrical).

\section{(a) Seismicity depth distribution}

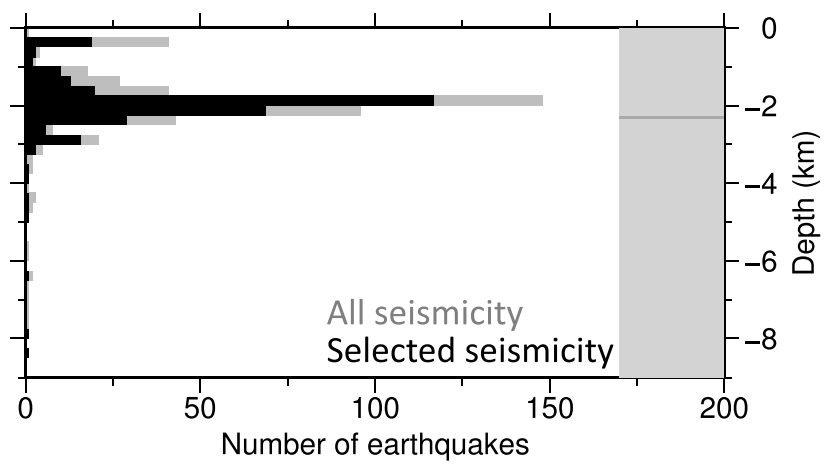

(b) Seismicity density planar distribution

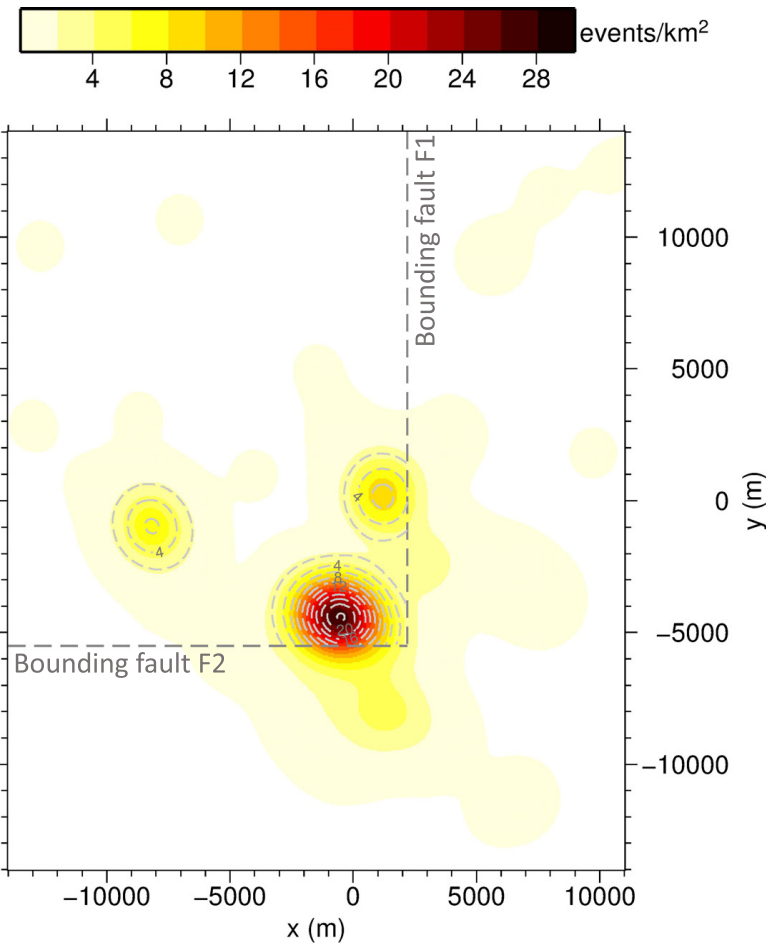

Figure 3. Spatial distribution of locally recorded microseismicity. (a) Depth distribution $(0.25 \mathrm{~km}$ bins width); the grey bars represent all seismicity while the black bars refer to the seismicity selected for our study. The position of the storage layer is also indicated (dark grey line on the right). (b) Planar distribution of seismicity density: the colours refer to the entire seismicity data set, while the overlapping contours ( 2 events $\mathrm{km}^{-2}$ intervals) refer to the selected seismicity. The location of the bounding faults from our model geometry is also indicated.

In our case study, the injection/extraction sources consist of multiple wells operating in two main zones $\left(S^{A}\right.$ and $S^{B}$ in Fig. 1b). In each of the two zones, a few months after the beginning of the operation, the injected gas forms a 'bubble' within the reservoir layer (the 'cushion gas'), over which additional gas is injected/extracted. The cushion gas volume at a specific time is the cumulative injected minus extracted gas, and it is needed to optimize seasonal storage operations. We modelled the volume of porous rock in which the cushion gas is contained at a certain time as a volumetric cylindrical source embedded in a water-saturated (reservoir) layer (hereafter 'gas source volume'). Pressure changes of the volumetric source are 
(a) 3D sketch

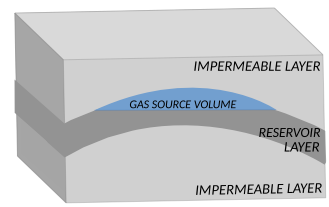

(c) Axisymmetric model

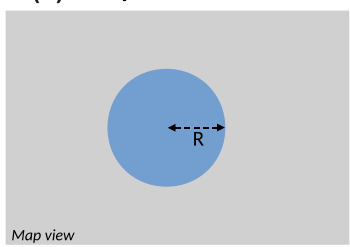

(d) One-boundary model

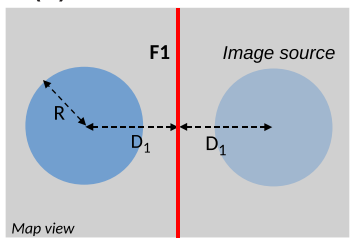

(b) Model section view

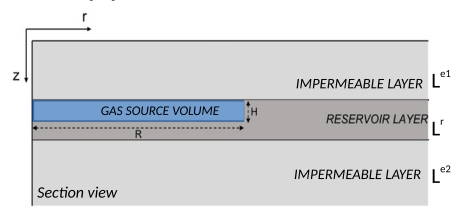

(e) Two-boundaries model

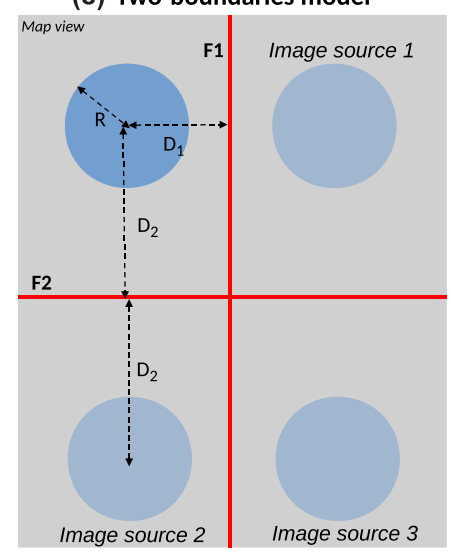

Figure 4. Schematic representation of the model implementation. 3-D model sketch (a), model section view (b), and map views of the model with no (c), one (d) and two (e) boundaries. The blue circles represent the cylindrical source, the shaded blue circles represent the image sources, and the red lines represent the vertical impermeable boundaries.

due to gas injection and extraction rates (see Figs $2 \mathrm{a}$ and $\mathrm{b}$ ). We kept fixed the source height ( $H=35 \mathrm{~m}$, half of the reservoir thickness), and we estimated its radius $\mathrm{R}$ proportionally to the cushion gas volume at the beginning of the analysed period. The approximation of using a source with fixed dimensions will be discussed in Section 5. Fig. 4 shows a 3-D sketch, and section and map views of the model geometry.

We first implemented two separate models for each of the two injection areas $\mathrm{S}^{\mathrm{A}}$ and $\mathrm{S}^{\mathrm{B}}$. For both, we considered the same simplified 3-layers structure representing the reservoir layer $\left(\mathrm{L}^{\mathrm{r}}\right)$ sealed between two layers of very low permeability and characterized by identical poroelastic parameters: the upper layer $\left(\mathrm{L}^{\mathrm{e} 1}\right)$ and the lower half-space $\left(\mathrm{L}^{\mathrm{e} 2}\right)$. Fig. 4(b) shows a section view of the model: the reservoir layer $\mathrm{L}^{\mathrm{r}}$, located at a depth of $\sim 2300 \mathrm{~m}$, has a thickness of $70 \mathrm{~m}$, that is the 60 per cent NTG (net-to-gross ratio) of the total reservoir layer thickness $(118 \mathrm{~m})$. The layers depth values are summarized in Table 1.

To model the effect of vertical impermeable faults bounding the reservoir and acting as a barrier for the fluid diffusion, we used the images method: a linear combination of unbounded single-source POEL solutions that satisfies the no-flux boundary condition on a vertical plane. This is obtained by superposing a mirror source solution to the real source (Fig. 4d). With the same technique, we developed the model to address the simultaneous presence of two perpendicular vertical impermeable faults, which is relevant for our case study (Fig. 4e).

It has to be noted that this method satisfies the boundary condition only for Darcy's flux. Therefore, the solution near the boundary is well approximated in the assumption that the coupling between the deformation and pore-pressure diffusion is negligible (i.e. the porepressure induced by the deformation is small and vice versa). The fully coupled solution with a vertical impermeable fault bounding the reservoir cannot be addressed with POEL. In fact, POEL uses semi-analytical solutions that are valid only in axisymmetric configurations, and the axial symmetry is inevitably lost in the presence of a vertical planar boundary.

The following scheme describes the main steps we followed to obtain a complete solution for the pore-pressure distribution and time-series for two simultaneously active sources with two boundaries.

1 Single source, one-boundary model: In order to simulate the effect of the lateral bounding fault F1, for each source separately we added a new, identical source mirrored from the boundary. The distances between the source centers and the boundary $\left(\mathrm{D}_{1}{ }^{\mathrm{A}}\right.$ and $\mathrm{D}_{1}{ }^{\mathrm{B}}$, for $\mathrm{S}^{\mathrm{A}}$ and $\mathrm{S}^{\mathrm{B}}$, respectively) have been chosen according to the distance between the injection wells groups and fault F1. Their values are displayed in Table 2.

2 Single source, two-boundaries model: In order to simulate the effect of the second fault F2, a second boundary is needed. In general, if there is more than one boundary, additional image sources are required (e.g. Ferris et al. 1962). When two boundaries are intersecting, the number of required image sources depends on the angle between the boundaries, with the simplest case being perpendicular boundaries requiring three image sources ( $c f$. further discussion in Section 5). Given the fault position in our case study (Fig. 1b), perpendicular boundaries represent a reasonable approximation. The distances between the source centers and the second boundary $\left(\mathrm{D}_{2}{ }^{\mathrm{A}}\right.$ and $\mathrm{D}_{2}{ }^{\mathrm{B}}$, for $\mathrm{S}^{\mathrm{A}}$ and $\mathrm{S}^{\mathrm{B}}$, respectively, Table 2) have been chosen according to the distance between the injection wells groups and fault F2.

3 Two-sources, two-boundaries model: Finally, to obtain a complete solution including both sources, considering the poroelastic model linearity, we superpose the two-boundaries solutions for $\mathrm{S}^{\mathrm{A}}$ and $\mathrm{S}^{\mathrm{B}}$, with a distance between the sources $\mathrm{D}_{2}{ }^{\mathrm{A}}-\mathrm{D}_{2}{ }^{\mathrm{B}}=3500 \mathrm{~m}$.

\subsection{Pore-pressure model: parametric study}

In addition to the geometry setup, the model needs the definition of the medium material properties. In particular, POEL software requires the definition of five parameters for each layer: the shear modulus $(\mu)$, the Poisson's ratio under drained and undrained conditions $\left(v\right.$ and $\left.v_{u}\right)$, the Skempton coefficient $(B)$, and the hydraulic diffusivity $(D)$.

For our case study, the availability of geomechanical models and in situ measurements provide useful information to constrain the values of $\mu, v$ and approximate values of porosity $(\phi)$ for all layers (cf. Table 1). Therefore, we only need to constrain $v_{u}\left(v \leq v_{u} \leq 0.5\right)$, $B(0 \leq B \leq 1)$ and $D$.

For the external layers, we used the formula for the Skempton coefficient (Skempton 1954)

$B=\frac{1}{1+\frac{\phi K}{K_{f}}}$

This equation links B to the bulk modulus of the soil structure $K=2 \mu(1+v) /[3(1-2 v)]$, that of the fluid $K_{f}(\sim 2.2 \mathrm{GPa}$ for water $)$ and the porosity $\phi$. We obtained $B^{\mathrm{Le}} \sim 0.5$.

In order to constrain $v_{u}$, we used the relation linking $B$ to $v, v_{u}$ and $\alpha$, the dimensionless coefficient of effective stress $0 \leq \alpha \leq 1$, (eq. 3 in Kümpel 1991)

$B=\frac{3\left(v_{u}-v\right)}{(1-2 v)\left(1+v_{u}\right) \alpha}$

Assuming $B^{\mathrm{Le}}=0.5$, this equation provides a tighter range for the undrained Poisson's ratio: $v_{u}^{\mathrm{Le}}=0.28(\alpha \rightarrow 0)$ and $v_{u}^{\mathrm{Le}}=0.4$ 
Table 1. Parameter values of the different poroelastic model layers. Italic blue font indicates the values known a priori; underlined grey font the ones kept fixed, and bold red font the ranges used for the parametric study.

\begin{tabular}{|c|c|c|c|c|c|c|c|}
\hline Layer & Depth (m) & $\begin{array}{l}\text { Shear modulus } \mu \\
\text { (GPa) }\end{array}$ & $\begin{array}{l}\text { Poisson's } \\
\text { ratio, } v\end{array}$ & Porosity, $\phi$ & $\begin{array}{c}\text { Undrained } \\
\text { Poisson's ratio, } v_{u}\end{array}$ & $\begin{array}{l}\text { Skempton's } \\
\text { coefficient, B }\end{array}$ & $\begin{array}{l}\text { Diffusivity } D \\
\left(\mathrm{~m}^{2} \mathrm{~s}^{-1}\right)\end{array}$ \\
\hline$\overline{\mathrm{L}^{\mathrm{e} 1}}$ & $0-2260$ & 25 & 0.28 & 5 per cent & 0.4 & 0.5 & $10^{-5}$ \\
\hline$L^{r}$ & $2260-2340$ & 21 & 0.28 & 10 per cent & $0.28 \leq \overline{v_{u}}{ }^{\mathrm{L} r} \leq 0.5$ & $0 \leq \overline{B^{\operatorname{Lr}}} \leq 1$ & $0.1 \leq \bar{D}^{\mathrm{Lr}} \leq 12$ \\
\hline $\mathrm{L}^{\mathrm{e} 2}$ & $2340-\infty$ & 25 & 0.28 & 5 per cent & 0.4 & 0.5 & $\underline{10^{-5}}$ \\
\hline
\end{tabular}

Table 2. Features of the two cylindrical model sources $\mathrm{S}^{\mathrm{A}}$ and $\mathrm{S}^{\mathrm{B}}$. Values of the distance between the source center and the vertical boundaries $\mathrm{F} 1$ and $\mathrm{F} 2$ and of the source radius for the time intervals $\mathrm{T} 1, \mathrm{~T} 2$ and $\mathrm{T} 3$.

\begin{tabular}{lccccc}
\hline & $\begin{array}{c}\text { Distance-Source centre to } \\
\text { boundary F1 }\end{array}$ & $\begin{array}{c}\text { Distance-Source centre to } \\
\text { boundary F2 }\end{array}$ & $\begin{array}{c}\text { Source } \\
\text { radius-Interval T1 }\end{array}$ & $\begin{array}{c}\text { Source } \\
\text { radius-Interval T2 }\end{array}$ & $\begin{array}{c}\text { Source } \\
\text { radius-Interval T3 }\end{array}$ \\
\hline Source $\mathrm{S}^{\mathrm{A}}$ & $\mathrm{D}_{1}{ }^{\mathrm{A}}=2200 \mathrm{~m}$ & $\mathrm{D}_{2}{ }^{\mathrm{A}}=5500 \mathrm{~m}$ & $\mathrm{R}_{\mathrm{T} 1}{ }^{\mathrm{A}}=1500 \mathrm{~m}$ & $\mathrm{R}_{\mathrm{T} 2}{ }^{\mathrm{A}}=1600 \mathrm{~m}$ & $\mathrm{R}_{\mathrm{T} 3}{ }^{\mathrm{A}}=1700 \mathrm{~m}$ \\
Source $\mathrm{S}^{\mathrm{B}}$ & $\mathrm{D}_{1}{ }^{\mathrm{B}}=1400 \mathrm{~m}$ & $\mathrm{D}_{2}{ }^{\mathrm{B}}=2000 \mathrm{~m}$ & $\mathrm{R}_{\mathrm{T} 1}^{\mathrm{B}}=700 \mathrm{~m}$ & $\mathrm{R}_{\mathrm{T} 2}{ }^{\mathrm{B}}=850 \mathrm{~m}$ & $\mathrm{R}_{\mathrm{T} 3}{ }^{\mathrm{B}}=1090 \mathrm{~m}$ \\
\hline
\end{tabular}

$(\alpha=1)$. We selected the latter value in order to represent the higherend configuration. As discussed thoroughly in the Supplementary Material (Section S1), the effect of a variation of the $v_{u}^{\text {Le }}$ value within this range does not cause a significant variation of the model results.

The diffusivity is related to $B, v, v_{\mathrm{u}}$, the permeability, $k$, and the dynamic fluid viscosity, $\eta_{f}$, through the equation (eq. 46 in Kümpel 1991)

$D=\frac{2}{9} \frac{(1-v)\left(1+v_{u}\right)^{2}}{\left(1-v_{u}\right)\left(v_{u}-v\right)} \frac{k}{\eta_{f}} \mu B^{2}$.

Since the external layers represent aquitards that seal the reservoir layer, we impose a typically very low value for the permeability $k^{\mathrm{Le}}=0.0002 \mathrm{md}\left(1\right.$ millidarcy $\left.=10^{-15} \mathrm{~m}^{2}\right)$. We assumed $\eta_{f}=0.5 \times 10^{-3} \mathrm{Pas}$, that is the dynamic viscosity of water at temperature $\sim 50{ }^{\circ} \mathrm{C}$, at $\sim 2 \mathrm{~km}$ depth (i.e. considering a gradient of $25^{\circ} \mathrm{C} \mathrm{km}^{-1}$, while ignoring year-round average surface temperature variations of $\sim 10^{\circ} \mathrm{C}$ since they do not cause significant variations of our parameters estimation). Using the previously described values for $B^{\mathrm{Le}}, v^{\mathrm{Le}}, v_{\mathrm{u}}^{\mathrm{Le}}$, we therefore obtained a diffusivity of $D^{\mathrm{Le}}=10^{-5}$ $\mathrm{m}^{2} \mathrm{~s}^{-1}$ for the external layers.

For the reservoir layer $\mathrm{L}^{\mathrm{r}}$, we know the values of $\mu^{\mathrm{Lr}}=21 \mathrm{GPa}$, $\nu^{\mathrm{Lr}}=0.28$ and $\phi^{\mathrm{Lr}} \sim 10$ per cent that, analogously to the external layers, could be used to roughly constrain the unknown parameters $B^{\mathrm{Lr}}, D^{\mathrm{Lr}}, v_{u}{ }^{\mathrm{Lr}}$. However, the model results strongly depend on these parameters. To investigate the sensitivity of the solutions with respect to different parameter combinations (and model configurations, i.e. with and without boundaries), we performed an extensive parametric study to better constrain $B^{\mathrm{Lr}}, D^{\mathrm{Lr}}$ and $v_{u}{ }^{\mathrm{Lr}}$ by comparing estimated and measured pore-pressure variations. In particular, we compared the time-series of pore-pressure measures at the monitoring wells to the model results obtained varying the most uncertain poroelastic parameters of the reservoir layer. Since POEL does not provide the solution within the source volume, we used a conservative approach and considered the modelled pore-pressure as an average of the results within the source radius at a depth just below the source, inside the reservoir layer $(z=2325 \mathrm{~m})$. The resulting time-series of the modelled pore-pressure were then compared to the observed pore-pressure values.

To perform the parametric study, we selected a time interval of 240 d between August 2015 and April 2016, including 3-injection/3extraction episodes (T1 interval, yellow stripe in Fig. 2), and we considered the pore-pressure variations referenced to the pore-pressure value of the first day of injection (Fig. 5). We chose this interval because it is located at about $3 \mathrm{yr}$ from the onset of the operation, when
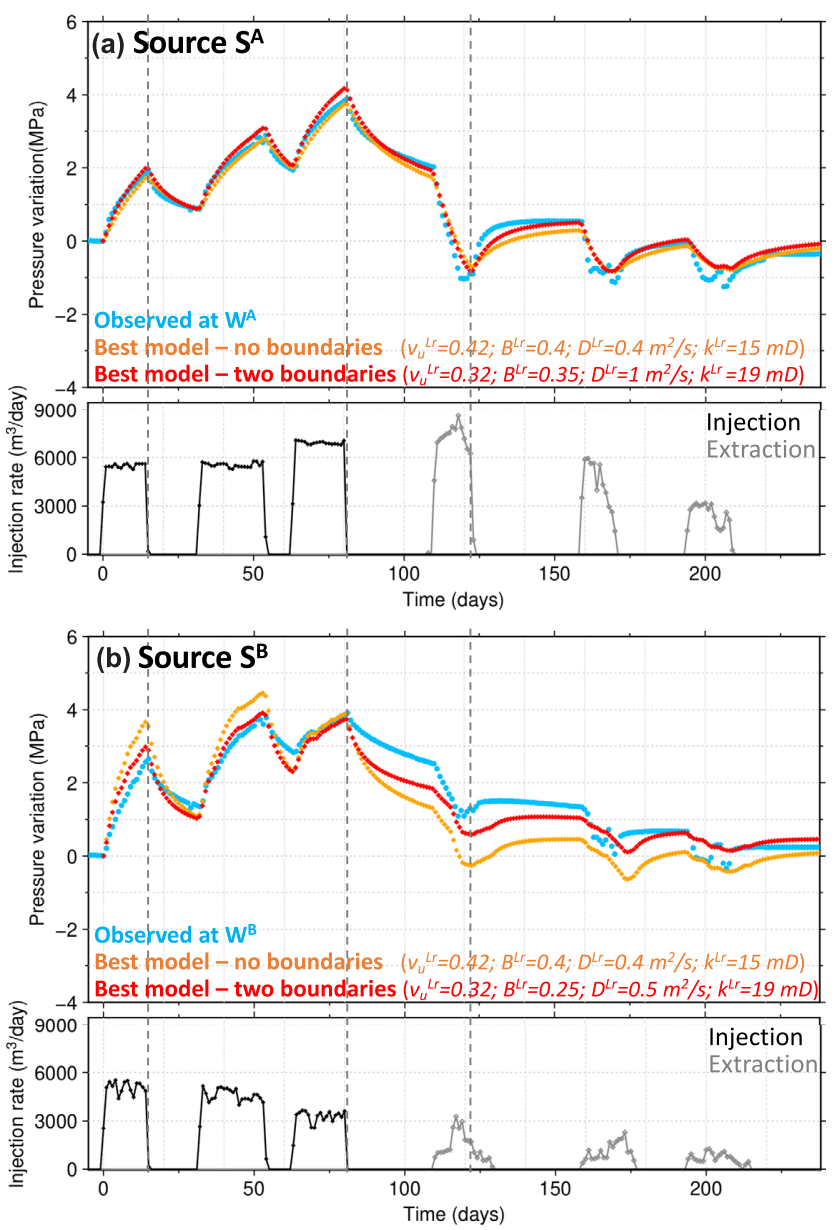

Figure 5. Observed (blue) and best-fitting modelled (orange and red) porepressure variation time-series for sources $\mathrm{S}^{\mathrm{A}}$ (a) and $\mathrm{S}^{\mathrm{B}}$ (b) in time interval T1 (cf. Fig. 2). The orange and red lines correspond respectively to the bestfitting one-source model configurations without and with two-boundaries (corresponding parameter values in brackets). The bottom panels show the injection (black) and extraction (gray) rate time-series. The vertical dashed lines depict the epochs at which pore-pressure distribution snapshots are shown in Figs 8 and S3 (days 15, 81 and 123).

the gas volume in place is large compared with the injected/extracted volumes (the maximum daily injection/production amount in $\mathrm{T} 1$ is about 0.5 per cent (for $\mathrm{S}^{\mathrm{A}}$ ) and 1 per cent (for $\mathrm{S}^{\mathrm{B}}$ ) of the cushion gas volume and the total volume variation in $\mathrm{T} 1$ is about 10 per 
cent (for $\mathrm{S}^{\mathrm{A}}$ ) and 40 per cent (for $\mathrm{S}^{\mathrm{B}}$ ) of the cushion gas volume). Also, T1 starts after a relatively long pause from the previous injection/production cycle $(\sim 110 \mathrm{~d})$, so that pressure diffusion processes already re-established the equilibrium within the aquifer layer.

We produced more than 100 pore-pressure models by varying the values of $B^{\mathrm{Lr}}, D^{\mathrm{Lr}}$ and $v_{u}{ }^{\mathrm{Lr}}$. In particular, we tested different values of the undrained Poisson's ratio within the interval $0.28 \leq$ $v_{u}^{\mathrm{Lr}} \leq 0.5$, and varied $B^{\mathrm{Lr}}$ between 0 and 1 and $D^{\mathrm{Lr}}$ up to $12 \mathrm{~m}^{2} \mathrm{~s}^{-1}$. The parameter values of the different layers are listed in Table 1: we indicate in blue the values known a priori, in gray the ones previously evaluated and kept fixed, and in red the range over which $B^{\mathrm{Lr}}, D^{\mathrm{Lr}}, v_{u}^{\mathrm{Lr}}$ have been varied.

We performed this parametric study for both $\mathrm{S}^{\mathrm{A}}$ and $\mathrm{S}^{\mathrm{B}}$ sources separately and, in each case, for both the axisymmetric case and the case with two-perpendicular boundaries.

\subsection{Seismicity model}

To investigate the seismicity-triggering potential of the estimated pore-pressure variation, we used the toolbox developed by Richter et al. (2020). This implements a seismicity model based on the common assumption that static stress changes lead to earthquake nucleation. Assuming the Coulomb stress as the parameter describing the stress state, increasing/decreasing Coulomb stress promotes/inhibits seismicity. Coulomb stress is defined as

$C F S=\tau-f \sigma_{e f f}=\tau-f\left(\sigma_{n}-p\right)$.

Here $\tau$ is the shear stress in the fault-slip direction, and $f$ is the coefficient of friction. $\sigma_{\text {eff }}$ is the effective compressive normal stress (defined as positive) on the fault plane given by the Cauchy stress acting on the rock skeleton $\left(\sigma_{n}\right)$ reduced by the pore-pressure $(p)$. Because the rupture mechanism is not known for the observed events and shear/normal stress cannot be resolved, we only considered the effect of pore-pressure changes assuming the proportionality $C F S$ $\sim p$. We therefore tested if the changes in pore-pressure associated with the UGS activity could explain the observed seismicity.

In our model, the friction is governed by rate-and-state dependency following the constitutive law derived by Dieterich (1994) for the evolution of seismicity on a population of faults under stress perturbation. The seismicity rate $R$ is a function of stressing history and given by

$R=\frac{r}{\dot{\tau} \gamma}$.

Here $r$ is the background seismicity rate, $\dot{\tau}$ the background shear stress rate, and $\gamma$ a state variable depending on the stressing history. For small changes in normal stress compared to the absolute values (i.e. lithostatic stress on the fault), the evolution in time $(t)$ of $\gamma$ is given by

$\mathrm{d} \gamma=\frac{\mathrm{d} t-\gamma \mathrm{d} S}{A \sigma_{n}}$.

with $S=\tau-f^{\prime} \sigma_{\text {eff }}$ and $f^{\prime}=f-\delta . A$ and $\delta$ are positive nondimensional constitutive parameters (Linker \& Dieterich 1992). $S$ therefore corresponds to the Coulomb stress with a reduced friction coefficient. In our application of the Coulomb Rate and State (RS) model, $S$ is assumed simply proportional to the pore-pressure $(S \sim p)$.

In addition to the RS-model, the toolbox provides the implementation of the linear Coulomb failure model (CM) and the Poisson model for comparison. The Poisson model describes temporally and spatially random seismicity, such as the one expected for a constant - in space and time - tectonic stressing rate $\left(R=r_{0}\right)$. Conversely, the CM-model considers, as the RS-model, a dependence of the earthquake rate on the UGS-induced pressure changes in addition to the constant background seismicity rate. However, the CM-model assumes that the seismicity rate is proportional to the pore-pressure variations related to an instantaneous earthquake triggering. In contrast, the response of the previously described RS-model is delayed and nonlinear.

In the basic CM-model, faults are critically loaded, and every positive stress change causes seismic activity with a seismicity rate of

$R\left(\boldsymbol{x}_{\boldsymbol{n}}, t_{k}\right)=r_{0}+a \dot{S}\left(\boldsymbol{x}_{\boldsymbol{n}}, t_{k}\right) \cdot H\left(\dot{S}\left(\boldsymbol{x}_{\boldsymbol{n}}, t_{k}\right)\right)$.

Here stress and seismicity rates are calculated on a spatial grid of $N$ points with coordinates $\boldsymbol{x}_{\boldsymbol{n}}=\left(x_{n}, y_{n}, z_{n}\right) \quad(n=1, \ldots, N)$ and at $K$ time steps $t_{k}(k=1, \ldots, K)$. The parameter $a$ is a proportionality factor, and $H$ is the Heaviside function with $H\left(\dot{S}\left(\boldsymbol{x}_{\boldsymbol{n}}, t_{k}\right)\right)=1$ for $\dot{S}\left(\boldsymbol{x}_{\boldsymbol{n}}, t_{k}\right) \geq 0$ and $H\left(\dot{S}\left(\boldsymbol{x}_{\boldsymbol{n}}, t_{k}\right)\right)=0$ otherwise. The dot indicates the time derivative that is performed over discrete (daily) time intervals.

We also implemented an alternative CM-model, hereafter defined as $\mathrm{CM}_{\text {sub }}$, in which a threshold-stress $\left(\Delta S_{0}\right)$ has to be reached at a given location before the seismicity rate starts evolving proportionally to the stress changes.

Unlike the RS-model, the CM-models do not intrinsically consider the Kaiser effect (Kaiser 1950; Lavrov 2003), i.e. seismicity can occur at a certain location only if stress exceeds the previously reached values at that location. Therefore, we also implemented CM-models accounting for the Kaiser effect $\left(\mathrm{CM}_{\mathrm{K}}\right.$ and $\left.\mathrm{CM}_{\text {sub_K }}\right)$, which implies adding a further condition to eq. (7)

$$
\begin{aligned}
R\left(\boldsymbol{x}_{\boldsymbol{n}}, t_{k}\right)= & r_{0}+a \dot{S}\left(\boldsymbol{x}_{\boldsymbol{n}}, t_{k}\right) \cdot H\left(\dot{S}\left(\boldsymbol{x}_{\boldsymbol{n}}, t_{k}\right)\right) \\
& \times H\left(S\left(\boldsymbol{x}_{\boldsymbol{n}}, t_{k}\right)-\max S\left(\boldsymbol{x}_{\boldsymbol{n}}, t<t_{k}\right)\right) .
\end{aligned}
$$

Since the depth distribution of the observed local seismicity is peaked at about $2 \mathrm{~km}$ depth, which is consistent with the storage layer depth within the seismicity location error of $1 \mathrm{~km}$ (see Fig. 3a), we restricted our seismicity analysis to a plane within the storage layer (at fixed depth $z=2325 \mathrm{~m}$ ).

For all the models, the seismicity rates $R\left(x_{n}, y_{n}, t_{k}\right)$ are calculated on the same horizontal spatial grid (steps of $50 \mathrm{~m}$ in both directions, with a planar extent of $16200 \mathrm{~m} \times 19450 \mathrm{~m}, n=1, \ldots, N$ with $N=126750$ ) and temporal sampling (daily) of the modelled pore-pressure variation data $p\left(x_{n}, y_{n}, t_{k}\right)$. The $R\left(x_{n}, y_{n}, t_{k}\right)$ values are separately calculated for each spatial grid point based on the pore-pressure variation distribution $p\left(x_{n}, y_{n}, t_{k}\right)$, assuming spatially uniform model parameters. However, due to the simplified POEL geometry, at the aim of the seismicity models evaluation and parameters estimation, we finally consider only the spatially summed rate $R\left(t_{k}\right)=\sum_{n=1}^{N} R\left(x_{n}, y_{n}, t_{k}\right)$. This is fitted to the observed event times $\left(t_{i}, i=1, \ldots, Z\right)$ using the maximum likelihood method (Daley \& Vere-Jones 2003; Hainzl et al. 2010). In particular, the $\log$-likelihood $\log L$ is given by

$\log L=\sum_{i=1}^{Z} \log \left(R\left(t_{i}\right)\right)-\sum_{k=1}^{K} R\left(t_{k}\right) \Delta t$.

The $\log L$-value is maximized with regard to the model parameters. Here the background rate $\left(r_{0}\right)$ is a model parameter for all the tested seismicity models (Poisson, $\mathrm{CM}, \mathrm{CM}_{\text {sub }}, \mathrm{CM}_{\mathrm{K}}, \mathrm{CM}_{\text {sub_K }}$ and RS). Further free parameters are the proportionality factor $a$ for the models $\mathrm{CM}, \mathrm{CM}_{\text {sub }}, \mathrm{CM}_{\mathrm{K}}$ and $\mathrm{CM}_{\text {sub_K }}$ and additionally the stress 


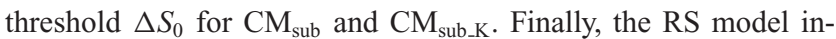
cludes as free parameters $A \sigma_{n}$ and the relaxation time $t_{a}=A \sigma_{n} / \dot{\tau}$ in addition to $r_{0}$.

Because the models involve a different number of free parameters, we used the Akaike Information Criterion, AIC $=2\left(M_{f}-\log L\right)$, to compare the model performances, with $M_{f}$ being the number of the free model parameter. The model providing the smallest AIC performs best.

\section{RESULTS}

\subsection{Parametric study results and effect of impermeable boundaries}

We computed the misfit for the $\mathrm{S}^{\mathrm{A}}$ source (both axisymmetric and two-boundaries configurations) calculated as the root mean square error (RMSE) between the modelled (average over the source area) and measured (at well $\mathrm{W}^{\mathrm{A}}$ ) pore-pressure time-series (Fig. 6). Each panel refers to a different value of the undrained Poisson's ratio, the $x$-axis represents $B^{\mathrm{Lr}}$, and the $y$-axis represents $D^{\mathrm{Lr}}$. The corresponding misfit plots for the $\mathrm{S}^{\mathrm{B}}$ source are shown in Fig. S2. The best-fitting parameters and corresponding RMSE values are provided in Table $\mathrm{S} 1$ (a)-(d).

All the analysed configurations reveal similar features and consistent preferred parameter values (associated with lower RMSE). In particular, a clear trade-off emerges between $B^{\mathrm{Lr}}$ and $D^{\mathrm{Lr}}$ with the low misfit area bending towards lower $B^{\mathrm{Lr}}$ and higher $D^{\mathrm{Lr}}$ with decreasing $v_{u}{ }^{\mathrm{Lr}}$. We note a slight preference for lower values of $B^{\mathrm{Lr}}$ and $D^{\mathrm{Lr}}\left(D^{\mathrm{Lr}}<3 \mathrm{~m}^{2} \mathrm{~s}^{-1}\right.$ and $\left.0.2<B^{\mathrm{Lr}}<0.6\right)$ because higher values of $D$ cause faster pore-pressure decay, which results in worse agreement with the observations.

Using eq. (3), for each value of $v_{u}^{\mathrm{Lr}}$ we calculated the permeability $k^{\mathrm{Lr}}$ corresponding to the different combinations of $B^{\mathrm{Lr}}$ and $D^{\mathrm{Lr}}$ (contours in Fig. 6 and Fig. S2). The best-fitting area approximately follows the $k^{\mathrm{Lr}}$ contour shape and is consistently bounded between $20 \mathrm{md}<k^{\mathrm{Lr}}<60 \mathrm{md}$ in all cases. Therefore, the permeability value is well constrained and lies within the range of values estimated with the petrophysical modelling provided by the company $\left(10 \mathrm{md}<k^{\mathrm{Lr}}\right.$ $<200 \mathrm{md}$ ).

By using the relations from Skempton (1954) and Kümpel (1991) (eqs 1 and 2 in Section 3.2), we can add further constraints on the best-fitting values that show trade-off. Eq. (1) gives $B^{\mathrm{Lr}} \sim 0.35$, which falls in the best-fitting range of our parametric study. Using eq. (2), $B^{\mathrm{Lr}}=0.35$ results in a preferred range for the undrained Poisson's ratio of $0.28 \leq v_{u}{ }^{\mathrm{Lr}} \leq 0.35$, while it is $0.28 \leq v_{u}{ }^{\mathrm{Lr}} \leq 0.4$ using $0.2 \leq B^{\mathrm{Lr}} \leq 0.6$.

The comparison between the observed and modelled porepressure time-series in interval T1 is shown in Fig. 5. The modelled time-series correspond to the best-fitting parameters combination for each source and configuration (axisymmetric and with two-boundaries, the corresponding parameters are indicated in the figure and highlighted in bold font in Table S1a-d). Despite the inevitable simplifications and assumptions of the model (e.g. cylindrical source geometry, fixed volume for gas in-place, three horizontal layers), we obtain a good fit for the observations (RMSE between 0.2 and $0.8 \mathrm{MPa}$ ). Besides, the modelled pore-pressure next to the bounding faults in both the axisymmetric and two-boundaries cases shows a higher-pressure build-up in the two-boundaries configuration (Fig. 7).

To assess the effect of adding impermeable vertical boundaries in the model, we consider the unbounded axisymmetric case as the reference configuration for our parametric study. Our outcomes show that similar best-fitting poroelastic parameters result in both configurations, with values in agreement with the petrophysical modelling results provided by the company. Furthermore, for both $\mathrm{S}^{\mathrm{A}}$ and $\mathrm{S}^{\mathrm{B}}$ sources, a notably better fit is achieved in the twoboundaries configuration (compare RMSE values of panels a and b in Fig. 6 and Fig. S2 and Table S1a-d). This improvement is particularly enhanced for source $\mathrm{S}^{\mathrm{B}}$, for which the RMSE decreases by about 50 per cent. $S^{B}$ is located closer to both bounding faults (Fig. 1b) and, even if a lower amount of gas is injected/extracted compared to the $\mathrm{S}^{\mathrm{A}}$ source, the observed pore-pressure time-series show a slower temporal decay after injection/extraction and a higherpressure build-up (Fig. 5). Thus, the presence of the bounding faults and the consequent pressure accumulation cause greater effects at this location.

Starting from the sets of best-fitting parameters previously identified for the separated sources, we ran models for the two-sources configuration, both with and without boundaries. In this case we estimated the RMSE considering pore-pressure time-series for both $\mathrm{S}^{\mathrm{A}}$ and $\mathrm{S}^{\mathrm{B}}$, obtaining $\mathrm{RMSE}=0.47 \mathrm{MPa}$ (with $v_{u}{ }^{\mathrm{Lr}}=0.35 ; B^{\mathrm{Lr}}=0.4$; $D^{\mathrm{Lr}}=0.8 \mathrm{~m}^{2} \mathrm{~s}^{-1} ; k^{\mathrm{Lr}}=19 \mathrm{md} ; c f$. Table S1e) in the case without boundaries and RMSE $=0.51 \mathrm{MPa}\left(\right.$ with $v_{u}{ }^{\mathrm{Lr}}=0.42 ; B^{\mathrm{Lr}}=0.35$; $D^{\mathrm{Lr}}=0.4 \mathrm{~m}^{2} \mathrm{~s}^{-1} ; k^{\mathrm{Lr}}=20 \mathrm{md} ; c f$. Table S1e) in the case with two boundaries. Fig. 8 displays the pore-pressure spatial distribution in the reservoir layer, right below the gas source volume $(z=2325 \mathrm{~m})$, corresponding to the latter best-fitting parameters at three different times in interval $\mathrm{T} 1(15,81$ and $123 \mathrm{~d})$; the corresponding plot in the no-boundaries configuration is shown in Fig. S3. The first two epochs are located at the end of two 15-20 d injection phases, while the third follows a $15 \mathrm{~d}$ extraction phase. While the radial outward (after injection) and inward (after extraction) pore-pressure diffusion is visible in both configurations, the presence of the vertical impermeable boundaries - and, in part, the superimposition of the two sources - causes a clear variation of the pore-pressure distribution with an evident pressure build-up (up to four times higher) along the boundaries.

\subsection{Estimation of seismicity rate and comparison with observed seismicity}

Using the results for the spatiotemporal distribution of pore-pressure variations from the POEL model (Section 4.1), we investigated the relation between the observed local seismicity and gas storage activities. In particular, in order to have longer time-series and account for the effect of pore-pressure accumulation with time, we ran further POEL simulations on two subsequent time intervals T2 and T3 (orange and green vertical stripes in Fig. 2, respectively). T2 includes 6-injections/3-extraction episodes during $330 \mathrm{~d}$ between April 2016 and February 2017, whereas T3 includes 10-injections/3-extraction episodes during 370 d between February 2017 and February 2018. Analogously to T1, we considered the pore-pressure variations for each interval T2 and T3 relative to the first day of injection. We simulated pore-pressure variations using the two-source and twoboundaries model configurations by modifying the sources radii according to the actual volume of cushion gas (Table 2). As for the poroelastic parameters, we used the best-fitting parameters previously found for the time interval T1 (Section 4.1 and Table S1e). The POEL model results in a good time-series fit $(\mathrm{RMSE}=0.44 \mathrm{MPa}$ for $\mathrm{T} 2$ and $\mathrm{RMSE}=0.72 \mathrm{MPa}$ for T3), confirming the robustness of the parametric study results. 
(a) Axisymmetric configuration
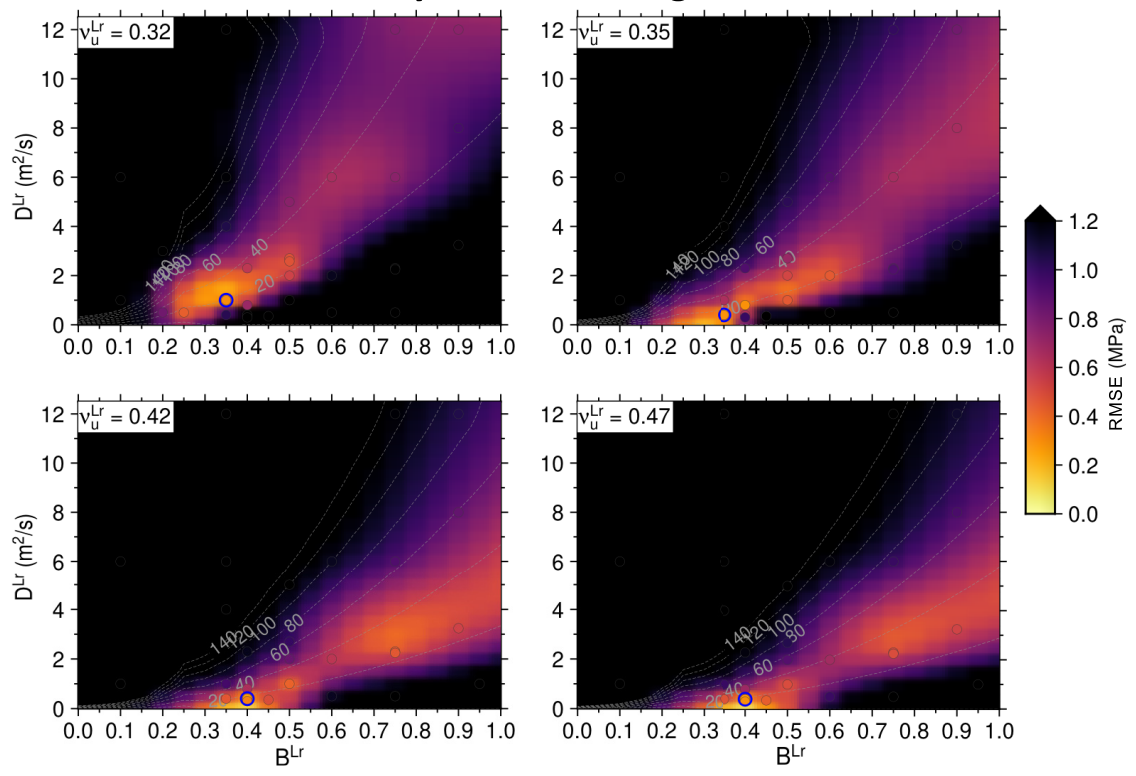

(b) Two-boundaries configuration
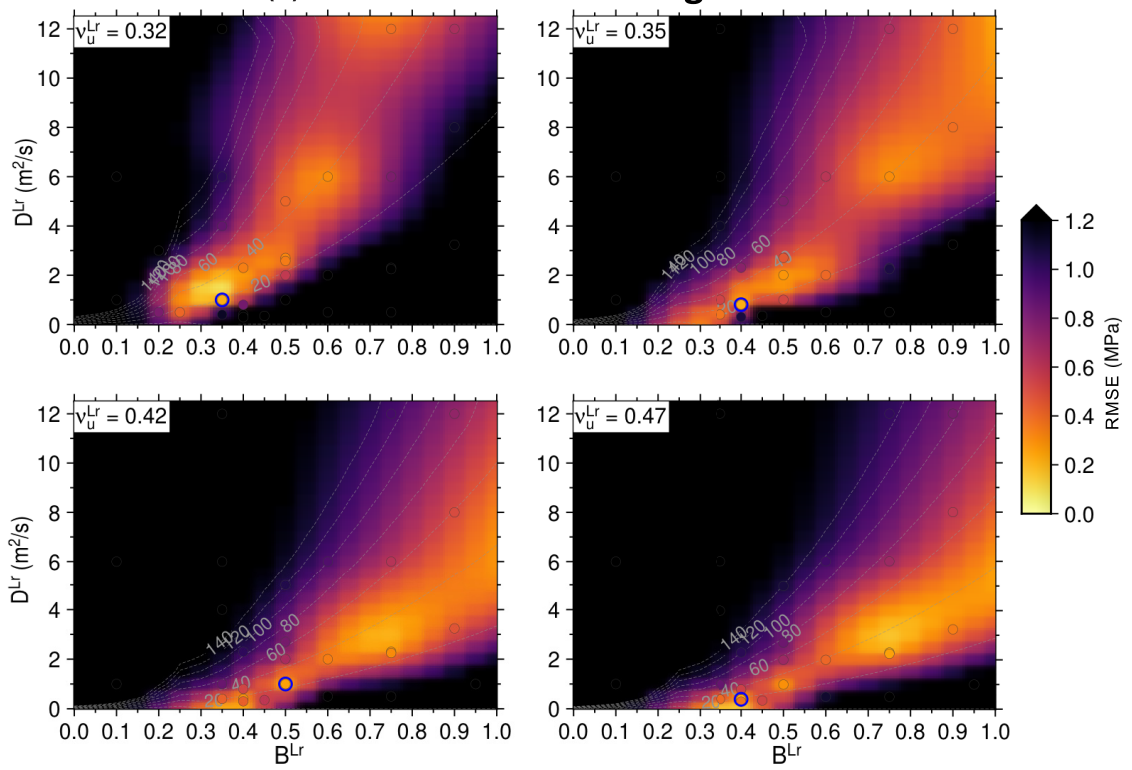

Figure 6. Misfit results for source $\mathrm{S}^{\mathrm{A}}$. RMSE (colour-coded, best fit as blue circle) is calculated between the modelled pore-pressure and the measured pore-pressure at well $\mathrm{W}^{\mathrm{A}}$ for different combinations of $B^{\mathrm{Lr}}, D^{\mathrm{Lr}}$ and $v_{u}^{\mathrm{Lr}}$. Open circles indicate the tested combinations of parameters values. Dashed contours delineate permeability $\left(k^{\mathrm{Lr}}\right)$ values. (a) Results for the axisymmetric configuration (no boundaries). (b) Results for the two-boundaries configuration.

Analogously to Figs 8 and S3, Fig. S4 shows the pore-pressure spatial distribution in the reservoir layer, right below the gas source volume $(z=2325 \mathrm{~m})$, at two times in interval T2 (130 and $235 \mathrm{~d}$ after first day of injection in $\mathrm{T} 2$, indicated as blue vertical dotted lines in Fig. 2). In this case too, the presence of boundaries causes pressure accumulation (up to four times higher than the unbounded model).

For our seismicity study, we selected the events with $M>$ $M c=0.0$ and, since we are considering the direct effect of porepressure diffusion on seismicity triggering, we considered only the events located within the area of the reservoir model geometry bounded by F1 and F2. Fig. 2(c) (orange bars) shows the resulting selected seismicity rate time-series (daily earthquake number), including 289 events. The corresponding depth and planar distributions are displayed, respectively, in Figs 3(a) (black bars) and 3(b) (dashed gray contour lines).

We estimated the seismicity model parameters using the modelled pore-pressure variation for the cases with and without impermeable boundaries and considering all time intervals (T1, T2 and T3, $940 \mathrm{~d}$ in total). This complete time-series has been obtained by joining the pore-pressure time-series estimated for each interval. The resulting pore-pressure time-series corresponding to different locations within the reservoir layer are shown in Fig. 9 (top panels).

Even if the local seismic network was installed during T1 (in October 2015, $87 \mathrm{~d}$ after the beginning of T1-gray dashed line in Fig. 2), in order to account for the complete stressing history, we 

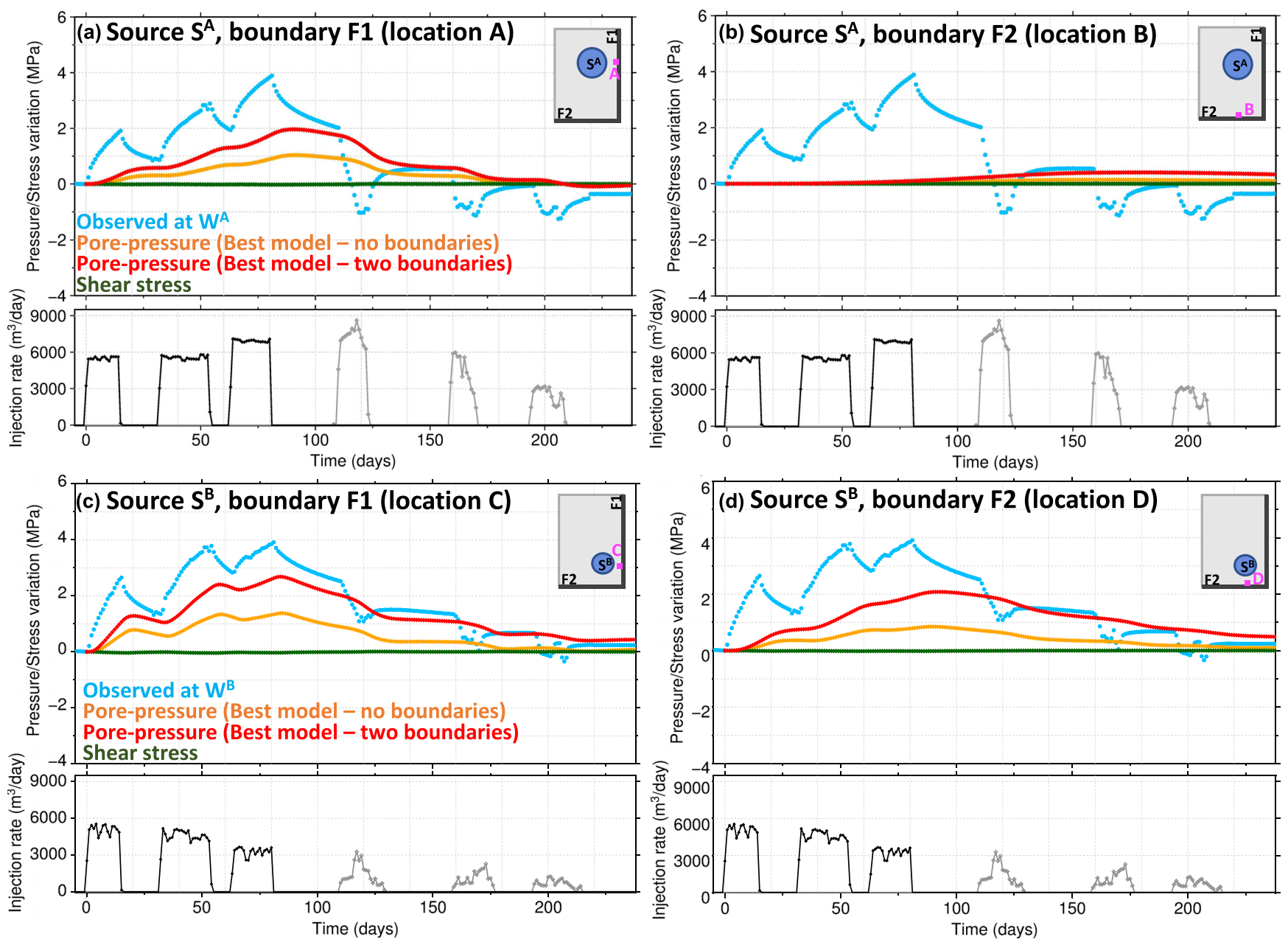

Figure 7. Comparison between times-series (interval T1, $c f$. Fig. 2) of modelled pore-pressure and shear stress variation at locations near to F1 (a, c) and F2 (b, d) boundaries (as indicated in the corresponding model sketch insets). The modelled pore-pressure (red and orange lines) refer to the best-fitting models for the $\mathrm{S}^{\mathrm{A}}(\mathrm{a}, \mathrm{b})$ and $\mathrm{S}^{\mathrm{B}}(\mathrm{c}, \mathrm{d})$ sources. The shear stress (green line) has been computed from the results of no-boundaries model setting. The observed pore-pressure time-series at the source locations (blue line) and the injection (black) and extraction (gray) rate time-series are also shown for reference.

considered the estimated pore-pressure variations for the entire T1 interval, including the first injection phase. However, for the sake of model parameters estimation, we considered only the seismicity rates after the seismic network installation.

The results - model parameters and AIC values — for the different seismicity models in both configurations (with and without impermeable boundaries) are listed in Table 3 . The AIC values show similar results in both configurations, with the RS-model performing best with comparable results for the $\mathrm{CM}_{\mathrm{K}}$ and $\mathrm{CM}_{\text {sub_K }}$ models. In contrast, the CM-models without the Kaiser effect are only slightly better than the Poisson model, indicating that the Kaiser effect plays an important role.

Fig. 10 shows the comparison between observed and model seismicity rates time-series $\left(\mathrm{RS}, \mathrm{CM}_{\mathrm{K}}\right.$ and $\mathrm{CM}_{\text {sub_K}}$ ) in the twoboundaries configuration (an analog plot for the case without boundaries is shown in Fig. S5). Since $\mathrm{CM}_{\mathrm{K}}$ and $\mathrm{CM}_{\text {sub_K }}$ perform almost identically, in the following we only discuss the RS and $\mathrm{CM}_{\mathrm{K}}$ models.

The Poisson model yields a constant rate of one event in three days $\left(r_{0}=0.3\right)$. Compared to this simple model, the others have lower background seismicity rates and estimate increased seismicity rates during injection periods (characterized by higher pore-pressure).
In particular, in the two-boundaries case, only 3 and 10 per cent of the events are related to background seismicity for $\mathrm{CM}_{\mathrm{K}}$ and RS, respectively. Therefore, the best models indicate a causal relation between the pore-pressure variations and the majority of the observed microearthquakes.

The RS seismicity rate time-series show a smooth increase during increasing pore-pressure phases, with the highest rates slightly above 2 events/day. The highest modelled seismicity rates occur during the main injection phases in T1 and T3, whereas lower seismicity rates in $\mathrm{T} 2$ result from smaller pore-pressure variations, in agreement with smaller peaks in the observed seismicity rates. Additionally, since the pressure level in T2 is lower than T1, the seismicity rates close to the injection sources in $\mathrm{T} 2$ are lower when the Kaiser effect is considered $\left(\mathrm{CM}_{\mathrm{K}}\right.$ and, implicitly in $\left.\mathrm{RS}\right)$. In our case the Kaiser effect indeed appears to prevent the nucleation of an event in the injection sources areas as long as the previous highest-pressure level is not exceeded.

Compared to the $\mathrm{CM}_{\mathrm{K}}$ seismicity rate time-series, the RS ones show a higher temporal correlation with the pore-pressure changes observed at the source area, with smooth increases and maxima corresponding in time with the occurrence of pore-pressure peaks, indicating a quick seismic response-within a day (Fig. 10). This better 

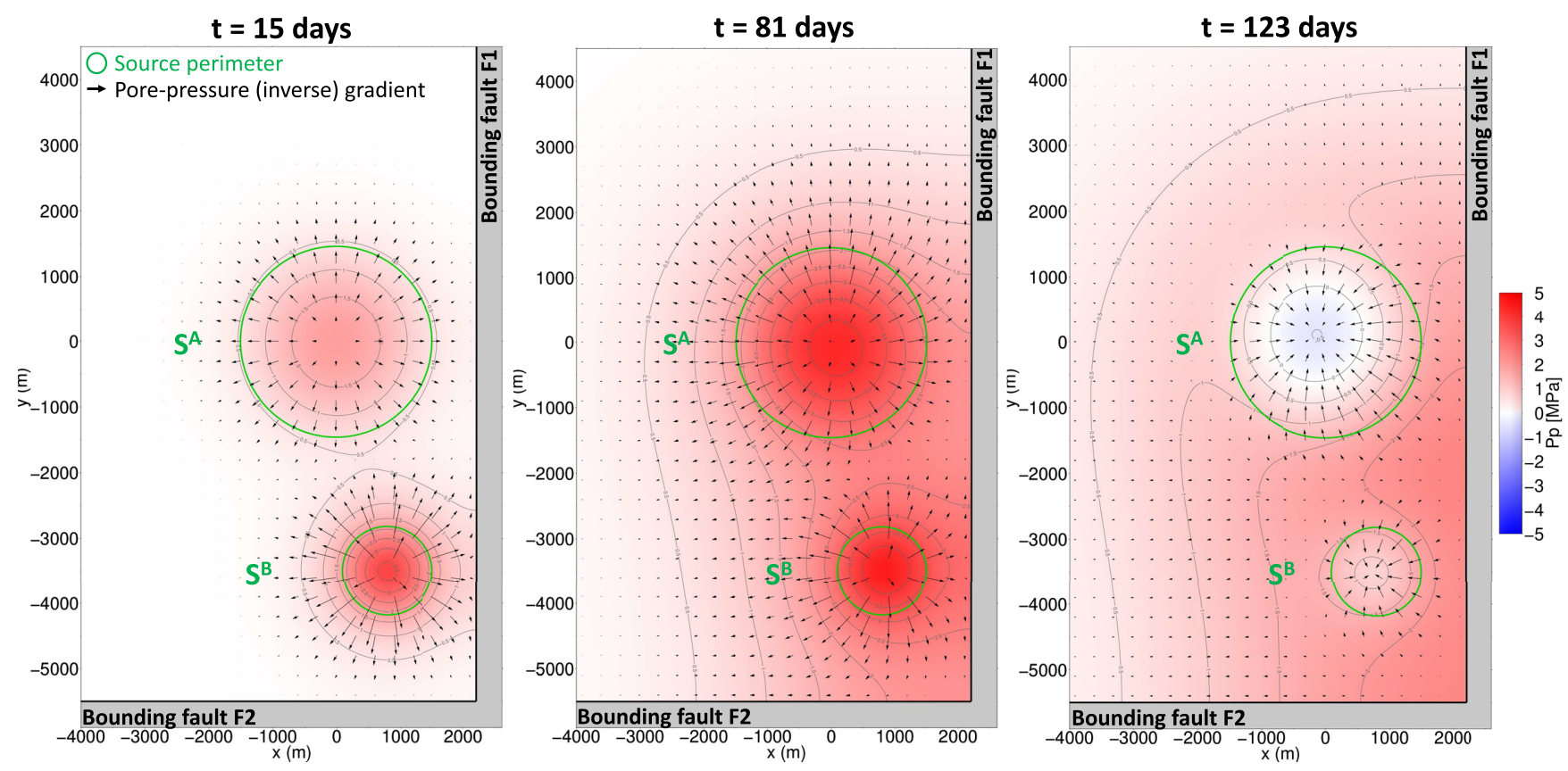

Figure 8. Snapshots (days 15, 81 and 123 of time interval T1, $c f$. Fig. 5) of the pore-pressure variation spatial distribution (colour-coded and contoured) on a $x-y$ plane right below the sources $(z=2325 \mathrm{~m})$ in the configuration with two-sources (green circles) and two-boundaries (gray stripes). The back arrows show the (inverse) pore-pressure gradient.

fit obtained by the RS model suggests that the coupling mechanism between pore-pressure change and seismicity is better represented by the nonlinear behaviour of the RS model.

Two epochs showing a significant underestimation of the observed seismicity (reaching maximum values on day 370, in T2: 8 events/day, and 883, in T3: 13 events/day, Fig. 10) correspond to clusters at F1, while most of the observed seismicity is generally located near F2. This observation possibly suggests that the two fault zones are characterized by different frictional conditions, that can also be linked to the differences in orientation of the faults in the regional tectonic setting. It is noteworthy furthermore that, in T2, the observed seismicity near F1 occurred at the onset of the injection, while, in T3, it occurred after the injection end when the production started (Fig. 10).

Fig. 9 (bottom plots) shows the comparison between the spatial distribution of the observed (contour lines) and modelled (RSmodel - colour-coded) seismicity (events $\mathrm{km}^{-2}$ ) cumulated over the whole studied period. Because the pore-pressure is initially higher at the injection locations, the Kaiser effect is more effective there, and the seismicity propagates away towards the faults. This is because the pore-pressure reaches the highest-pressure level close to the injection sources during the first injection period in T1. Therefore, the Kaiser effect appears to cause stress shadows close to the wells and a steady, slow increase of seismicity at larger distances.

When the impermeable boundaries are considered (Fig. 9b), the modelled seismicity is highest at the faults and their conjunction, providing a good fit with the observed seismic cluster locations, even though with lower density (up to $\sim 9$ events $\mathrm{km}^{-2}$ ). Conversely, the model without boundaries results in the highest seismicity located between the injection sources (Fig. 9a), suggesting that the introduction of the boundaries is essential to explain the spatial distribution of the observed seismicity.

Our seismicity model has spatially homogeneous parameters, that is it does not consider faults and lateral material heterogeneities, which could generate a more clustered seismicity and possibly provide a better fit with the observed seismicity (Richter et al. 2020). A way to account for this is to consider a larger location error of the observed seismicity. Assuming a standard deviation of $2 \mathrm{~km}$ (Fig. S6) leads to an observed seismicity density up to $\sim 7$ events $\mathrm{km}^{-2}$, that is in better agreement with the modelled one.

\section{DISCUSSION}

In this paper, we presented a POEL software application to an underground gas reservoir in Southern Europe with recorded seismic events. In particular, we addressed the spatiotemporal evolution of pore-pressure associated with injection/extraction of gas from multiple sources in an underground aquifer layer bounded by two faults.

Starting from the single-source (axisymmetric) solution for a horizontally layered poroelastic medium provided by POEL, and taking advantage of the linearity of the poroelastic problem, we applied the image method in order to satisfy the no-flux boundary condition on a vertical plane. We demonstrated how this method can be further extended by including an additional impermeable vertical plane perpendicular to the first one. Single-source solutions (either including the boundaries or not) can be linearly combined to assess the coexistence of multiple injection/extraction sources.

In order to analyse the sensitivity of the model results and the effect of the vertical boundaries, we performed an extensive parametric study over the unknown (or weakly constrained) poroelastic parameters of the reservoir layer by comparing modelled and observed pore-pressure variations. The results obtained for two different injection/extraction sources and with different model configurations (with and without boundaries) show consistent features. The model is sensitive to changes in the poroelastic parameters, providing a lower misfit for parameter values which are consistent in all the analysed configurations. 
(a) Without boundaries
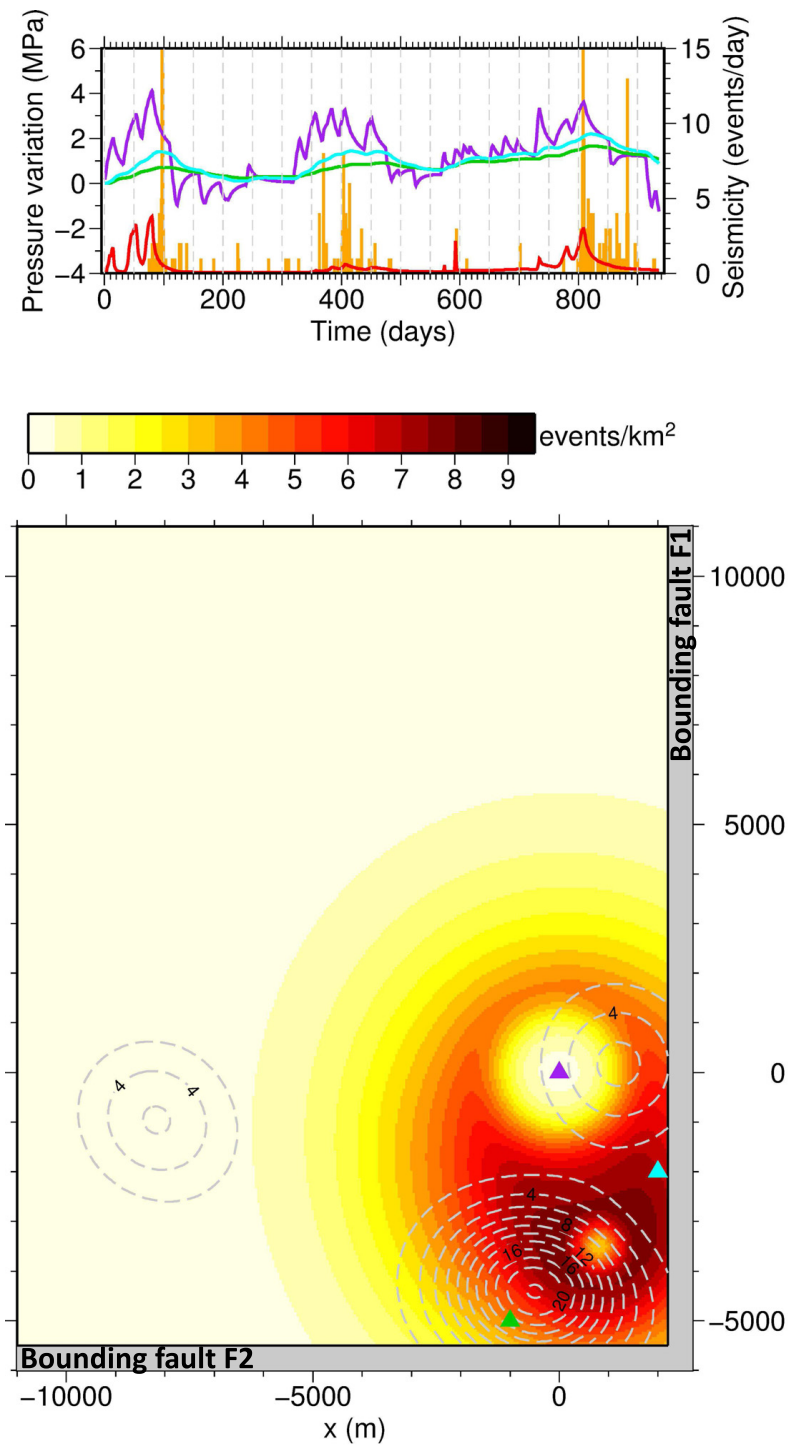

(b) With boundaries
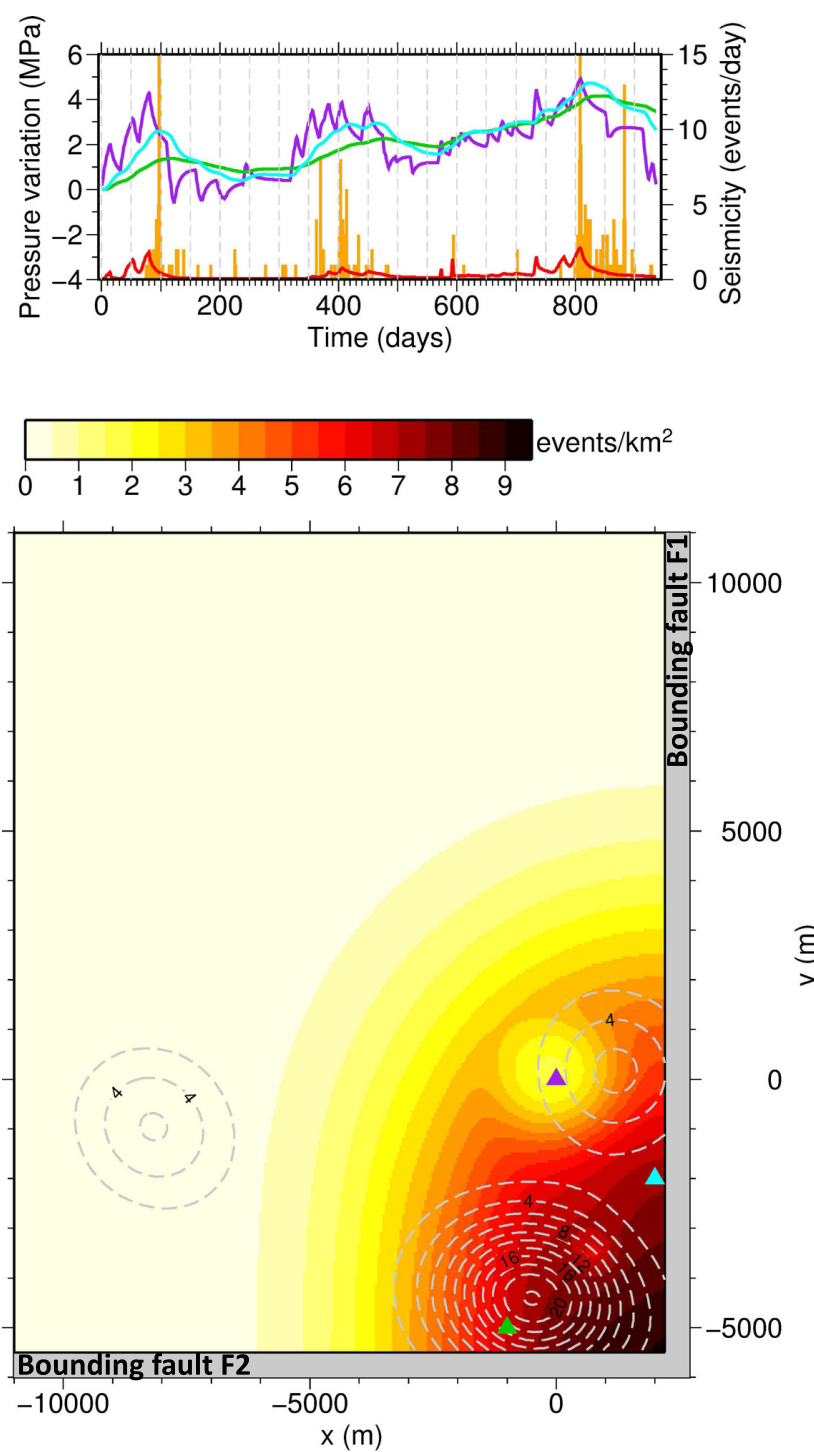

Figure 9. Seismicity model results. Map view of the cumulated modelled seismicity density (RS-model) for the model configurations (a) without and (b) with two impermeable boundaries. Gray contour lines show the observed seismicity density smoothed for a Gaussian location error with a standard deviation of $1 \mathrm{~km}$ (as in Fig. 3b). The top plots show the observed (orange) and modelled (red, RS-model) seismicity rates and the time-series of the estimated pore-pressure variation at the three locations indicated in the map (triangles with corresponding colours).

We were able to further constrain our model by exploiting information from a pre-existing geomechanical model, and using theoretical relations linking different poroelastic parameters. An improved estimate of all parameters could be achieved by comparing the modelled pore-pressure distribution with pore-pressure measurements at different distances from the injection areas, however such measurements were not available in our case. Furthermore, since our model considers a homogeneous and isotropic reservoir layer, the parameters values constrained through the parametric study should be considered as effective or averaged values over the aquifer layer.

We showed that the presence of two vertical impermeable boundaries provides an improvement of the fit with observations, and results in pore-pressure accumulation near the faults, especially for the source located closer to both of them $\left(\mathrm{S}^{\mathrm{B}}\right)$. This pore-pressure accumulation is potentially a key effect to help understand why seismicity possibly related to human activities occurs in certain preferential areas of the reservoir. Indeed, it has been proposed that the accumulation of pore-pressure adjacent to sealing faults causes pore-pressure gradients that, depending on the fault geometry, may induce seismicity (Chang \& Segall 2016). In addition, the presence of the bounding faults causes a variation of the temporal pattern in the pore-pressure diffusion (Figs 5 and 7) which may lead to a different modulation of micro-earthquakes, and thus affect the seismicity. Also, we observe that the presence of the bounding faults may generate differences in the stress gradient distribution: the unbounded model creates small wavelength pressure undulations, while the bounded models appear to generate a smoother field, although of higher stress (Figs 8, S3 and S4). The presence of bounding faults increases the stress level near the faults - and therefore the related seismicity, as also confirmed through the seismicity model - while without bounding faults the highest stress concentrates close to the injection sources. 
Table 3. Best-fitting parameters values and corresponding AIC results estimated for the different seismicity models.

\begin{tabular}{|c|c|c|c|c|}
\hline \multirow[t]{2}{*}{$\begin{array}{l}\text { Model-no } \\
\text { boundaries }\end{array}$} & \multicolumn{2}{|c|}{ Parameters } & \multirow[t]{2}{*}{ AIC } & \multirow{2}{*}{$\begin{array}{c}\Delta \mathbf{A I C} \\
\mathrm{AIC}_{\mathrm{X}}-\mathrm{AIC}_{\mathrm{Poisson}}\end{array}$} \\
\hline & $\begin{array}{c}\text { Background seismicity } r_{0} \\
\text { [events/day] }\end{array}$ & $\begin{array}{c}\left.a \text { [events } /\left(\mathrm{km}^{2} \mathrm{MPa}\right)\right] ; \Delta S_{0} \\
\left.[\mathrm{MPa}] ; A \sigma_{n}[\mathrm{MPa}] ; t_{a} \text { [days }\right]\end{array}$ & & \\
\hline Poisson & 0.33 & 1 & 1189 & / \\
\hline $\mathrm{CM}$ & 0.25 & $a=0.0005$ & 1176 & -13 \\
\hline $\mathrm{CM}_{\text {sub }}$ & 0.25 & $a=0.0006 ; \Delta S_{0}=0.0$ & 1176 & -13 \\
\hline $\mathrm{CM}_{\mathrm{K}}$ & 0.02 & $a=0.008$ & 867 & -321 \\
\hline $\mathrm{CM}_{\text {sub_K }}$ & 0.02 & $a=0.008 ; \Delta S_{0}=0.0008$ & 869 & -319 \\
\hline $\mathrm{RS}$ & 0.04 & $A \sigma_{n}=0.22 ; t_{a}=1.0 \times 10^{4}$ & 852 & -337 \\
\hline \multirow{2}{*}{$\begin{array}{l}\text { Model-with } \\
\text { boundaries }\end{array}$} & \multicolumn{2}{|c|}{ Parameters } & AIC & $\Delta \mathrm{AIC}$ \\
\hline & $\begin{array}{c}\text { Background seismicity } r_{0} \\
\text { [events/day] }\end{array}$ & $\begin{array}{c}\left.a \text { [events } /\left(\mathrm{km}^{2} \mathrm{MPa}\right)\right] ; \Delta S_{0} \\
\left.[\mathrm{MPa}] ; A \sigma_{n}[\mathrm{MPa}] ; t_{a} \text { [days }\right]\end{array}$ & & $\mathrm{AIC}_{\mathrm{X}}-\mathrm{AIC}_{\text {Poisson }}$ \\
\hline Poisson & 0.33 & 1 & 1189 & / \\
\hline $\mathrm{CM}$ & 0.18 & $a=0.0008$ & 1154 & -35 \\
\hline $\mathrm{CM}_{\text {sub }}$ & 0.18 & $a=0.0008 ; \Delta S_{0}=0.001$ & 1155 & -34 \\
\hline $\mathrm{CM}_{\mathrm{K}}$ & 0.01 & $a=0.004$ & 905 & -284 \\
\hline $\mathrm{CM}_{\text {sub_K }}$ & 0.01 & $a=0.004 ; \Delta S_{0}=0.0008$ & 907 & -282 \\
\hline $\mathrm{RS}$ & 0.03 & $A \sigma_{n}=0.28 ; t_{a}=6.0 \times 10^{3}$ & 906 & -283 \\
\hline
\end{tabular}

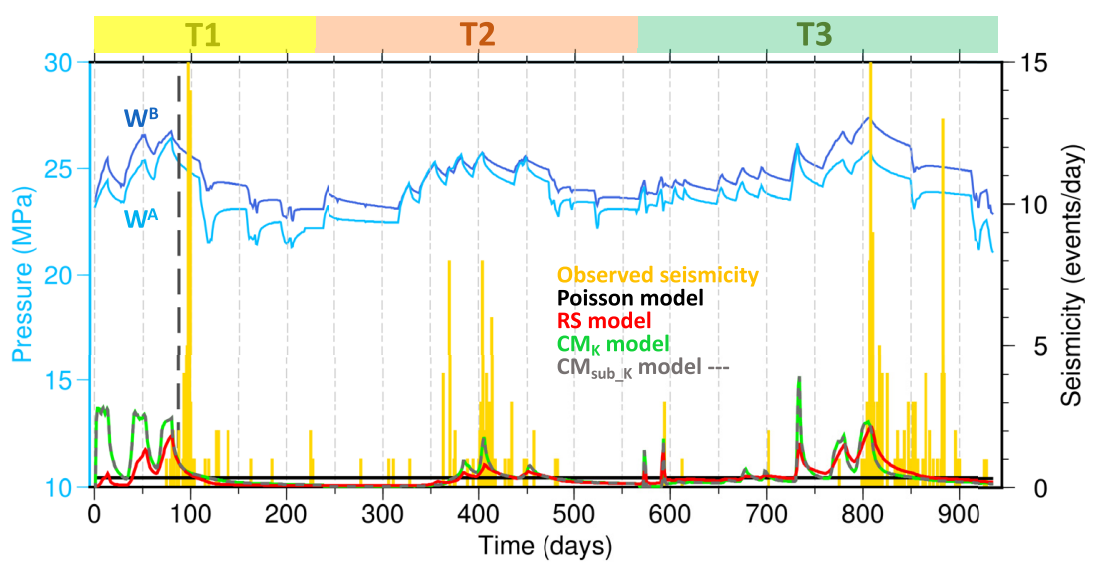

Figure 10. Comparison of best-modelled and observed seismicity rates for the model configuration with two impermeable boundaries. The black vertical dashed line marks the starting epoch of the time interval used for the seismicity model evaluation. The Poisson model result and measured pressure at wells $\mathrm{W}^{\mathrm{A}}$ and $\mathrm{W}^{\mathrm{B}}$ are also shown for reference.

As highlighted throughout the text, our method inevitably contemplates some assumptions and simplifications. Below, we provide a list of the main ones and discuss how they may affect our results and how we tried to mitigate their effects.

(i) Our model only accounts for single-phase fluids. This limitation requires using a volumetric source representing the volume of rock containing the gas bubble, which prevents modelling the pressure within it. However, this is not a critical point when, as in our study case, the focus is on the pore-pressure diffusion within the water-saturated aquifer. Furthermore, the diffusion of pressure in the gas is faster than in the water domain. The reason is the higher mobility of gas compared to water and the likely increase of permeability in the gas source volume due to increased porosity with pressure. Also, the effect of possible gas-fluid/fluid-gas transitions and their possibly strong effects on fluid compressibility remain still mostly unknown.

(ii) The gas source volume is fixed during the time-frame of a simulation. To overcome this limitation, we selected periods of limited duration $(\leq 1 \mathrm{yr})$ starting more than $3 \mathrm{yr}$ after the beginning of the UGS operations. Thus, the injected/extracted volumes are much smaller than the volume in place. In our case, in all the analysed time intervals, the maximum daily injection/production amount is about 0.5 per cent (for $\mathrm{S}^{\mathrm{A}}$ ) to 2 per cent (for $\mathrm{S}^{\mathrm{B}}$ ) of the cushion gas volume, and the cumulated volume variation within each interval is about 10 per cent (for $\mathrm{S}^{\mathrm{A}}$ ) to 40 per cent (for $\mathrm{S}^{\mathrm{B}}$ ) of the cushion gas volume.

(iii) The modelled pore-pressure values in the reservoir layer are only available outside the volumetric source, while the wells pressure is measured within the gas source volume. To overcome this limitation, we adopted a conservative approach considering pressure values averaged over a circle of the same radius and right below the source, as close as possible to the volumetric source, given the model spatial resolution.

(iv) Only vertical, planar impermeable boundaries can be implemented. Therefore, any dip angle of the bounding faults is neglected. 
Because of the limited reservoir layer thickness $(<100 \mathrm{~m})$, this approximation is not expected to have a large influence within the reservoir layer, where we focus our analysis.

(v) The boundary faults are assumed to be fully impermeable, which is not necessarily the case in nature. Our method may account for partially permeable faults by using different linear combinations of the mirror sources. However, given the uncertainties on fault permeability, and the good fit with observations, we just considered fully impermeable faults. This implies that the pore-pressure accumulation we compute near the boundaries represents a high-end estimate.

(vi) The consideration of vertical impermeable boundaries (image method) causes the loss of the coupling between pore-pressure and deformation solutions (Section 3.1). This limitation implies that only the pore-pressure variation, and not the stress, can be computed next to the boundaries, which may influence the Coulomb stress change computation. This problem cannot be easily overcome due to the way how the POEL solutions are intrinsically calculated. However, the pore-pressure change can be a good indicator of the Coulomb stress change, especially when the magnitude of porepressure variation is significantly larger than the shear stresses. The latter is the case in our study, where the shear stress variation at the bounding faults is up to 2 orders of magnitude smaller than the pore-pressure variation. This is shown in Fig. 7, where we display the time-series for the shear stress (green lines) and pore-pressure variations (orange lines) relative to the unbounded case at the same location (i.e. at the bounding faults, points A, B, C and D).

The application of the method of images becomes more complicated when multiple boundaries are involved, since more image sources are needed to prevent the flow diffusing from an image source to penetrate another boundary. However, the approach described here could be used in similar settings involving one or more quasi-vertical sealing faults. A typical case is a gas or oil field bounded by two quasi-parallel quasi-vertical faults (e.g. graben structures). In general, when there are two parallel, laterally unlimited boundaries, an infinite number of image sources is required. In practice, however, the solution can be well approximated using a finite number of image sources since it is only necessary to add image sources until the additional one would have a negligible influence on the sum of all image-source effects (e.g. Ferris et al. 1962). The number of image sources required for the solution to converge depends on various factors such as the time scale over which the solution is required, the diffusion process velocity, and the source-boundary distance. In Fig. S7, we provide an example of implementation of two parallel boundaries performed using the previously calculated pore-pressure solution for source $\mathrm{S}^{\mathrm{A}}$ in interval $\mathrm{T} 1$.

In the case of two intersecting boundaries, a solution can be found by adding a finite number of image sources depending on the angle between the boundaries. In particular, the most simple cases for the construction of closed image systems occur when the angle between the boundaries can be approximated to an aliquot part of $360^{\circ}$-for example, an angle of $45^{\circ}$ would require seven image sources (Ferris et al. 1962).

Potentially, by properly arranging the image sources, more complex cases with more than two intersecting boundaries can also be implemented (Ferris et al. 1962).

Our model for the spatiotemporal evolution of the pore-pressure changes allows the study of the possible relation with recorded seismic events in the area. We compared the observed seismicity rates close to the UGS to the modelled seismicity rates based on three different models. One model assumes the seismicity constant in space and time (Poisson model) to test if the observed seismicity could be explained simply as background seismicity. The other two models assume a relation to the anthropogenic activity testing linear (CM-type models) or non-linear (RS model) frictional behaviour.

In general, both CM and RS models have been applied to study induced seismicity in different contexts (Dempsey \& Suckale 2017; Langenbruch et al. 2018; Norbeck \& Rubinstein, 2018; Shapiro, 2018; Candela et al. 2019; Jiang et al. 2021). In our case, the $\mathrm{CM}_{\mathrm{K}} / \mathrm{CM}_{\text {sub_K }}$ and RS models can explain the observed microseismicity in the examined time period of $2.3 \mathrm{yr}$ significantly better than the Poisson and simple CM (i.e. without Kaiser effect) models. The probability of seismicity increases during injection periods with a nearly linear and instantaneous relation between pore-pressure increase and seismicity rate. Since the previous stressing history and nonlinear effects influence the seismicity, assuming a rate-and-state relation slightly improves the results. The analysis of the spatial distribution of the earthquakes suggests that the introduction of two impermeable faults in the pore-pressure model improves the estimation of the seismicity spatial distribution. Additionally, due to the Kaiser effect, little seismicity is expected close to the injection wells after the first injection cycle. A strong role of the Kaiser effect has been observed also in the context of seismicity associated with volcanic activity, such as at Kafla volcano (Heimisson et al. 2015).

Our seismicity model reproduces the observed seismicity rate trend, but not its amplitude, large fluctuations, and detailed timing. At least a part of this misfit can be ascribed to factors such as the simplifications of the pore-pressure model (e.g. simplified geometry, homogeneous material properties, impermeable boundaries), the fact that the interactions between earthquakes (e.g. aftershocks) are not implemented, and that the stressing history before the T1 period is not considered. Nonetheless, our results about the temporal and spatial seismicity distribution suggest that a part of the observed seismic patterns could be related to the UGS activity and possibly reveal different behaviours of the two bounding faults. More accurate models - and longer data time-series - are needed to confirm or reject this hypothesis.

The lower amount of seismicity that has been recorded beyond the bounding faults or farther from the injection sources might be related to additional triggering mechanism different from direct pore-pressure diffusion, such as elastic and fully coupled poroelastic stresses, that are able to transmit forces to great distances, well beyond the fluid pressure (e.g. Goebel et al. 2017; Goebel \& Brodsky 2018; Segall \& Lu 2015). Furthermore, based on the depth distribution and magnitude of the observed microseismicity $(M \approx 1)$, in our approach we only considered the pore-pressure change inside the aquifer layer as triggering mechanism for the seismicity. Some seismicity is however also observed at shallower and deeper depths in the adjacent layers (Fig. 3a). Here the pore-pressure variations are orders of magnitudes smaller, but the poroelastic stresses can give an important contribution in triggering seismicity (e.g. Deng et al. 2020; Jiang et al. 2021). The occurrence of seismicity just across the F2 boundary could also be ascribed to a not complete impermeability of the fault or the presence of a thick fault zone. Finally, at least a portion of the observed seismicity is most likely due to tectonic processes rather than human activities.

\section{CONCLUSIONS}

To study gas reservoirs embedded in poroelastic media, elaborated and detailed numerical modelling techniques are often applied, such as finite element models. Instead, we focused in this paper on the 
highly flexible and easily formulated POEL tool that can be applied to similar cases. We show the potential and limitations of the POEL code, that can be freely downloaded and is straightforward to use. Its flexibility enables to deal with the presence of multiple sources and impermeable boundaries. This method could also be used jointly with and propaedeutically to more complex and detailed fully numerical models to evaluate the effects of the involved parameters and of the impermeable boundaries. Being a semi-analytical model, it allows a better insight about the different factors contributing to the pore-pressure diffusion process. Parametric studies with POEL can provide useful prior knowledge for more realistic and complex 3-D models, and the coupling with seismicity models enables the exploration of the aleatoric and epistemic uncertainties for seismic hazard assessments.

\section{ACKNOWLEDGEMENTS}

We sincerely thank Hans-Joachim Kümpel and David Dempsey for the accurate review and precious comments. We thank our industrial partner Enagas Transporte S.A.U. for its contribution to this study.

We thank Eleonora Rivalta and her research group at GFZ for the helpful discussions.

This work was funded by the Bundesministerium für Bildung und Forschung (BMBF) in the framework of the project SECURE (Sustainable dEployment and Conservation of Underground Reservoirs and Environment, research grant 03G0872A) and by the Deutsche Forschungsgemeinschaft (DFG) in the framework of the project 'Modellierung von Schmelzaufstieg durch das Asthenosphaeren-Lithosphaeren-Kontinentale Krustensystem' (research grant RI 2782/5-1).

FS and FM designed the research framework, analysed the data, performed the poroelastic computations and wrote the original draft. GR performed the seismicity analysis and contributed to the original draft. BGC provided the data and contributed to the design and implementation of the research. RW, SH, and TD supervised the findings of this work. All authors discussed the results and contributed to the final manuscript.

Figures have been produced by using the Generic Mapping Tools (GMT) software (Wessel et al. 2013).

\section{DATA AVAILABILITY}

The background data for this study is owned by Enagas S.A.U. and cannot be disclosed without a prior written consent from the company.

POEL code can be freely downloaded at the GFZ Tool Development Lab website https://www.gfz-potsdam.de/en/section/physic s-of-earthquakes-and-volcanoes/inf rastructure/tool-development $-\mathrm{lab} /$.

\section{REFERENCES}

Altmann, J.B., Müller, T.M., Müller, B.I.R., Tingay, M.R.P. \& Heidbach, O., 2010. Poroelastic contribution to the reservoir stress path, Int. J. Rock Mech. Min, 47(7), 1104-1113.

Altmann, J.B., Müller, B.I.R., Müller, T.M., Heidbach, O., Tingay, M.R.P. \& Weißhardt, A., 2014. Pore-pressure stress coupling in 3D and consequences for reservoir stress states and fault reactivation, Geothermics, 52, 195-205.

Barbour, A.J., Norbeck, J.H. \& Rubinstein, J.L., 2017. The effects of varying injection rates in Osage County, Oklahoma, on the 2016 Mw 5.8 Pawnee Earthquake, Seismol. Res. Lett., 88(4), 1040-1053.
Biot, M.A., 1941. General theory of three-dimensional consolidation, $J$. Appl. Phys., 12, 155-164.

Candela, T. et al., 2019. Depletion-induced seismicity at the Groningen gas field: coulomb rate-and-state models including differential compaction effect, J. Geophys. Res., 124, 7081-7104.

Chang, K.W. \& Segall, P., 2016. Injection-induced seismicity on basement faults including poroelastic stressing, J. Geophys. Res., 121, 2708-2726.

Chang, K.W. \& Segall, P., 2017. Reduction of injection-induced porepressure and stress in basement rocks due to basal sealing layers, Pure appl. Geophys., 174, 2649-2661.

Daley, D. \& Vere-Jones, D., 2003. An introduction to the theory of point processes. in Elementary Theory and Methods, 2nd edn., Vol. I, Springer.

Dempsey, D. \& Suckale, J., 2017. Physics-based forecasting of induced seismicity at Groningen gas field, the Netherlands, Geophys Res. Lett., 44, 1-10.

Deng, K., Liu, Y. \& Chen, X., 2020. Correlation between poroelastic stress perturbation and multidisposal wells induced earthquake sequence in Cushing, Oklahoma, Geophys. Res. Lett., 47, e2020GL089366, doi:10.1029/2020GL089366.

Dieterich, J., 1994. A constitutive law for rate of earthquake production and its application to earthquake clustering, J. Geophys. Res., 99, 2601-2681.

Evans, K.F., Zappone, A., Kraft, T., Deichmann, N. \& Moia, F., 2012. A survey of the induced seismic response to fluid injection and geothermal and $\mathrm{CO}_{2}$ reservoirs in Europe, Geothermics, 41, 30-54.

Ferris, J.G., Knowles, D.B., Brown, R.H. \& Stallman, R.W., 1962. Theory of Aquifer Test, U. S. Geol. Surv. Water Supply Pap., 1536-E.

Foulger, G.R., Wilson, M.P., Gluyas, J.G., Julian, B.R. \& Davies, R.J., 2018. Global review of human-induced earthquakes, Earth Sci. Rev., 178, $438-$ 514.

Goebel, T.H.W., Weingarten, M., Chen, X., Haffener, J. \& Brodsky, E.E., 2017. The 2016 Mw5.1 Fairview, Oklahoma earthquakes: evidence for long-range poroelastic triggering at $>40 \mathrm{~km}$ from fluid disposal wells, Earth Planet. Sc. Lett., 472, 50-61,.

Goebel, T.H.W. \& Brodsky, E.E., 2018. The spatial footprint of injection wells in a global compilation of induced earthquake sequences, Science, 361(6405), 899-904.

Grigoli, F. et al., 2017. Current challenges in monitoring, discrimination, and management of induced seismicity related to underground industrial activities: a European perspective, Rev. Geophys., 55, 310-340.

Grünthal, G., 2014. Induced seismicity related to geothermal projects versus natural tectonic earthquakes and other types of induced seismic events in Central Europe, Geothermics, 52, 22-35.

Hainzl, S., Steacy, S. \& Marsan, D., 2010. Seismicity models based on Coulomb stress calculations, Community Online Resource for Statistical Seismicity Analysis. Available at: http://www.corssa.org, doi:10.5078/corssa-32035809.

Haug, C., Nüchter, J.A. \& Henk, A., 2018. Assessment of geological factors potentially affecting production-induced seismicity in North German gas fields, Geomech. Ener. Environ., 16, 15-31.

Heimisson, E.R., Einarsson, P., Sigmundsson, F. \& Brandsdóttir, B., 2015. Kilometer-scale Kaiser effect identified in Krafla volcano, Iceland, Geophys. Res. Lett., 42, 7958-7965.

Improta, L., Valoroso, L., Piccinini, D. \& Chiarabba, C., 2015. A detailed analysis of wastewater-induced seismicity in the Val d'Agri oil field (Italy), Geophys. Res. Lett., 42, 2682-2690.

Jahr, T., Jentzsch, G., Letz, H. \& Sauter, M., 2005. Fluid injection and surface deformation at the KTB location: modelling of expected tilt effects, Geofluids, 5, 20-27.

Jahr, T., Letz, H. \& Jentzsch, G., 2006. Monitoring fluid induced deformation of the earth's crust: a large scale experiment at the KTB location/Germany, J. Geodyn., 41(1-3), 190-197.

Jiang, G., Liu, L., Barbour, A.J., Lu, R. \& Yang, H., 2021. Physics-based evaluation of the maximum magnitude of potential earthquakes induced by the Hutubi (China) underground gas storage, J. Geophys. Res., 126, e2020JB021379, doi:10.1029/2020JB021379.

Jolley, S.J., Dijk, H., Lamens, J., Fisher, Q., Manzocchi, T., Eikmans, H. \& Huang, Y., 2007. Faulting and fault sealing in production simulation models: brent Province, northern North Sea, Petrol. Geosci., 13, 321-340. 
Jolley, S.J., Fisher, Q.J. \& Ainsworth, R.B., 2010. Reservoir compartmentalization: an introduction, Geol. Soc. Lond. Spec. Publ., 347, 1-8.

Kaiser, J., 1950. An investigation in the occurrence of noises in tensile tests or a study of acoustic phenomena in tensile tests, PhD thesis, Tech. Hoch. München, Germany.

Kettermann, M., Smeraglia, L., Morley, C.K., von Hagke, C. \& Tanner, D.C., 2020. Fault Sealing In Tanner, D., Brandes, C. (eds.) Understanding Faults - Detecting, Dating, and Modeling, Elsevier, pp. 283-350, doi:10.1016/B978-0-12-815985-9.00008-4.

Kümpel, H.-J., 1991. Poroelasticity: parameters reviewed, Geophys. J. Int., 105, 783-799.

Langenbruch, C., Weingarten, M. \& Zoback, M.D., 2018. Physics-based forecasting of man-made earthquake hazards in Oklahoma and Kansas, Nat. Commun., 9, 3946.

Lavrov, A., 2003. The Kaiser effect in rocks: principles and stress estimation techniques, Int. J. Rock Mech. Min. Sci., 40, 151-171.

Linker, M. \& Dieterich, J., 1992. Effects of variable normal stress on rock friction: observations and constitutive equations, J. Geophys. Res., 97, 4923-4940

Liu, W., Jiang, D., Chen, J., Daemen, J.J.K., Tang, K. \& Wu, F., 2018. Comprehensive feasibility study of two-well-horizontal caverns for natural gas storage in thinly-bedded salt rocks in China, Energy, 143, 1006-1019.

Norbeck, J.H. \& Rubinstein, J.L., 2018. Hydromechanical earthquake nucleation model forecasts onset, peak, and falling rates of induced seismicity in Oklahoma and Kansas, Geophys. Res. Lett., 45, 1-13.

O’Neill, S.R., Jones, S.J., Kamp, P.J.J., Swarbrick, R.E. \& Gluyas, J.G., 2018. Pore-pressure and reservoir quality evolution in the deep Taranaki Basin, New Zealand, Mar. Petrol. Geol., 98, 815-835.

Orlic, B., 2016. Geomechanical effects of $\mathrm{CO}_{2}$ storage in depleted gas reservoirs in the Netherlands: Inferences from feasibility studies and comparison with aquifer storage, J. Rock Mech. Geotech., 8(6), 846-859.

Pasala, S.M., Forster, C.B., Deo, M. \& Evans, J.P., 2013. Simulation of the impact of faults on $\mathrm{CO}_{2}$ injection into sandstone reservoirs, Geofluids, 13(3), 344-358.

Ricard, L.P., MacBeth, C., HajNasser, Y. \& Schutjens, P., 2012. An evaluation of pore-pressure diffusion into a shale overburden and sideburden induced by production-related changes in reservoir fluid pressure, J. Geophys. Eng., 9, 345.

Richter, G., Hainzl, S., Dahm, T. \& Zöller, G., 2020. Stress-based, statistical modeling of the induced seismicity at the Groningen Gas Field, The Netherlands, Environ. Earth Sci., 79, 252.

Shapiro, S.A., 2018. Seismogenic index of underground fluid injections and productions, J. Geophys. Res., 123, 7983-7997.

Shapiro, S.A., Kummerow, J., Dinske, C., Asch, G., Rothert, E., Erzinger, J., Kümpel, H.-J. \& Kind, R., 2006. Fluid induced seismicity guided by a continental fault: injection experiment of 2004/2005 at the German Deep Drilling Site (KTB), Geophys. Res. Lett., 33, L01309, doi:10.1029/2005GL024659.

Segall, P. \& Lu, S., 2015. Injection-induced seismicity: Poroelastic and earthquake nucleation effects, J. Geophys. Res., 120, 5082-5103.

Skempton, A.W., 1954. The pore-pressure coefficients A and B., Geotechnique, 4(4), 143-147.

Wang, R. \& Kümpel, H.-J., 2003. Poroelasticity: efficient modelling of strongly coupled, slow deformation processes in a multilayered half-space, Geophysics, 68(2), 705-717.

Wessel, P., Smith, W.H., Scharroo, R., Luis, J. \& Wobbe, F., 2013. Generic mapping tools: Improved version released, EOS, Trans. Am. Geophys. Un., 94(45), 409-410.

Wu, F., Gao, R., Zou, Q., Chen, J., Liu, W. \& Peng, K., 2020. Long-term strength determination and nonlinear creep damage constitutive model of salt rock based on multistage creep test: Implications for underground natural gas storage in salt cavern, Ener. Sci. Eng., 8, 1592-1603.

Yu, H., Harrington, R.M., Liu, Y. \& Wang, B., 2019. Induced seismicity driven by fluid diffusion revealed by a near-field hydraulic stimulation monitoring array in the Montney Basin, British Columbia, J. Geophys. Res., 124, 4694-4709.
Zbinden, D., Rinaldi, A.P., Urpi, L. \& Wiemer, S., 2017. On the physicsbased processes behind production-induced seismicity in natural gas fields, J. Geophys. Res., 122, 3792-3812.

Zhai, G., Shirzaei, M., Manga, M. \& Chen, X., 2019. Pore-pressure diffusion, enhanced by poroelastic stresses, controls induced seismicity in Oklahoma, P. Natl. Acad. Sci. USA, 116(33), 16 228-16 233.

\section{SUPPORTING INFORMATION}

Supplementary data are available at $G J I$ online.

Figure S1. Effect of $v_{u}^{\text {Le }}$ value variation. (a) RMSE (colour-coded, best fit as blue circle) is calculated between the modelled porepressure and the measured pore-pressure at well WA for different combinations of $B^{L r}$ and $D^{L r}$. The open circles indicate the tested parameters values combinations and the dashed contours delineate permeability $(k L r)$ values. (b) Observed (blue) and modelled (orange and red) pore-pressure variation time-series in time interval T1 (refer to Fig. 2 in the main text). The orange and red lines correspond, respectively, to the best-fitting model for the case $v_{u}{ }^{\text {Le }}=0.3$ and the respective model for the case $v_{u}{ }^{L e}=0.4$. The bottom panels show the injection (black) and extraction (gray) rate time-series.

Figure S2. Misfit results for source $S^{\mathrm{B}}$. RMSE (colour coded, best fit as blue circle) is calculated between the modelled pore-pressure and the measured pore-pressure at well WB for different combinations of $B^{L r}, D^{L r}$ and $v_{u}^{L e}$. Open circles indicate the tested parameters values combinations. Dashed contours delineate permeability $\left(k^{L r}\right)$ values. (a) Results for the axisymmetric configuration (no boundaries). (b) Results for the two-boundaries configuration.

Figure S3. Snapshots (days 15, 81 and 123 of the T1 interval, see Fig. 5 in the main text) of pore-pressure spatial distribution (colourcoded and contoured) on a $x-y$ plane right below the sources $(z=$ $2325 \mathrm{~m}$ ) in the configuration with two-sources (green circles) and no-boundaries (the dashed lines indicate the boundaries position as in Fig. 8 in the main text). The back arrows show the (inverse) pore-pressure gradient.

Figure S4. Snapshots (days 130 and 235 of the T2 interval, see blue vertical dotted lines in Fig. 2 of the main text) of pore-pressure spatial distribution (colour-coded and contoured) on a $x-y$ plane right below the sources $(z=2325 \mathrm{~m})$, in the configuration with two-sources (green circles) and (a) no-boundaries (the dashed lines indicate the boundaries position) and (b) two boundaries. The back arrows show the (inverse) pore-pressure gradient.

Figure S5. Comparison of best-modelled and observed seismicity rates for the model configuration without impermeable boundaries. The black vertical dashed line marks the starting epoch of the time interval used for the seismicity model evaluation. The Poisson model result and measured pressure at wells $\mathrm{W}^{\mathrm{A}}$ and $\mathrm{W}^{\mathrm{B}}$ are also shown for reference.

Figure S6. Seismicity model results. Map view of the cumulated modelled seismicity density (RS model) for the model configurations (a) without and (b) with two impermeable boundaries. Gray contour lines show the observed seismicity density smoothed for a Gaussian location error with standard deviation of $2 \mathrm{~km}$. The top plots show the observed (orange) and modelled (red) seismicity rates and the time-series of the estimated pore-pressure variation at the three locations indicated in the map (triangles with corresponding colours).

Figure S7. Example of two-parallel boundaries implementation. We used the previously computed solution for source $\mathrm{S}^{\mathrm{A}}$ in interval $\mathrm{T} 1$ to implement the effect of two parallel impermeable boundaries, both at a distance of $2000 \mathrm{~m}$ from the source center. (a) Sketch of the model implementation. The blue circles represent the cylindrical 
source, the shaded blue circles represent the image sources, and the red lines represent the vertical impermeable boundaries F1 and F2. (b) Results of pore-pressure variation time-series at the locations A and $\mathrm{B}$ indicated in (a) and localized in the aquifer layer right below the source $(z=2325 \mathrm{~m})$. Each line corresponds to increasing number of image sources as indicated in the legend. The results show that, in this case, three couples of image sources are sufficient for the solution to converge. The corresponding injection/production rate time-series are also represented (black/gray lines).

Table S1. Parametric study results. Each table collects the parameters and RMSE values associated to the best-fitting results for different configurations. Bold font highlights the lowest RMSE.
Table S1a. $\mathrm{S}^{\mathrm{A}}$ source, axisymmetric configuration (no boundaries). Table S1b. $\mathrm{S}^{\mathrm{A}}$ source, two-boundaries configuration.

Table S1c. $S^{B}$ source, axisymmetric configuration (no boundaries). Table S1d. $S^{B}$ source, two-boundaries configuration.

Table S1e. Configuration with both $\mathrm{S}^{\mathrm{A}}$ and $\mathrm{S}^{\mathrm{B}}$ sources. RMSE calculated using pore-pressure time-series at both sources.

Please note: Oxford University Press is not responsible for the content or functionality of any supporting materials supplied by the authors. Any queries (other than missing material) should be directed to the corresponding author for the paper. 